\title{
FIBER PRESERVING EQUIVALENCE
}

\author{
BY \\ RICHARD T. MILLER
}

\begin{abstract}
We give a theory of fibered regular neighborhoods based on a remarkable property of simplicial fibered projections. All the usual properties of regular neighborhoods are retained. Using Millett's fibered general position, together with the regular neighborhoods, we prove THEOREM. The simplicial space of codimension 4 PL embeddings of a complex into a PL manifold is lo. cally contractible at each point of the space of topological embeddings.
\end{abstract}

Introduction. Two embeddings $g_{1}, g_{2}$ of a space $X$ into a space $Q$ are equivalent if there is a homeomorphism $\Phi: Q \rightarrow Q$ such that $\Phi \circ g_{1}=g_{2}$. The local theory in the PL category is now understood. It is exposited in Edwards [E]. The positive results are summarized in

Edwards, Corollary 3'. Suppose $f: X \rightarrow$ int $Q$ is a TOP embedding of a compact polyhedron $X$ into a $P L$ manifold $Q$, and suppose $\operatorname{dim} X \leqslant \operatorname{dim} Q$ - 3. Then given any $\epsilon>0$, there is $a \delta>0$ such that any two PL embeddings of $X$ into int $Q$ which are within $\delta$ of $f$ are equivalent by an $\epsilon-P L$ ambient isotopy.

In codimensions less than three the corollary is false. In fact, it fails even if the $\epsilon$-ambient isotopy condition is dropped.

The corollary above is the same as the statement that the simplicial space of codimension 3 PL embeddings of a complex in a PL manifold is locally zero connected at points of the space of TOP embeddings. In this paper we prove that all the higher local homotopy groups of the space of codimension 4 embeddings vanish. This is the fibered close equivalence theorem. (See Theorem 1.) The previous best result was by Lusk [Lu]. He showed local $k$-connectivity of codimension 3 embeddings for $k \leqslant \operatorname{dim} Q-\operatorname{dim} X-3$.

The proof of Theorem 1 requires working in the context of the product $\boldsymbol{Q} \times \boldsymbol{A}$ where $\boldsymbol{Q}$ is a PL manifold and $\boldsymbol{A}$ is a simplicial complex. All procedures are designed to preserve the sets of the form $Q \times\{a\}, a \in A$. We signal this idea by the words fiber preserving, or fibered. En route to Theorem 1 we require fibered versions of most of the standard tools of PL topology. Some of

Received by the editors May 23, 1973.

AMS (MOS) subject classifications (1970). Primary 57C35.

Key words and phrases. Piecewise linear, embedding, isotopy. 
these are easy and we give proofs (e.g. simplicial approximation). Others automatically work in the fibered case (e.g. Alexander's trick). The important cone unknotting theorem follows from the nonfibered case [L], the fibered Alexander trick, and the covering $n$-isotopy theorem (see [H]). Millett [Mi, §3] does the general position theorem (see $\S 2$, Proposition 9, this paper for the statement). He also proves a fibered sunny collapsing theorem [Mi, §4].

Our principal contribution concerns fibered regular neighborhoods. The problem in defining such neighborhoods is illustrated by the fact that there is a fiber preserving PL homeomorphism $h$ of $R^{n} \times B^{k}$ to itself that fixes $0 \times$ $B^{k}$ and $R^{n} \times \partial B^{k}$ and such that $h\left(B^{n} \times B^{k}\right)$ does not fiber preserving PL ambient isotop to $B^{n} \times B^{k}$ fixing $0 \times B^{k} \cup R^{n} \times \partial B^{k}$. Otherwise, PL disc bundles would be the same as PL $R^{n}$ bundles. This is not the case [Bro].

We state one piece of philosophy and get two results. The philosophy is that one should always work in triangulations of $Q \times A$ that project simplicially to a triangulation of $A$. (If $f: Z \times A \rightarrow Q \times A$ is fiber preserving, it is always possible to make both $f$ and the projection of $Q \times A$ to $A$ simplicial simultaneously.)

Suppose $K$ and $L$ are two triangulations of $R^{1} \times B^{1}$ that agree on $R^{1} \times$ $\partial B^{1} \cup\{0\} \times B^{1}$ and project simplicially to the triangulation of $\{0\} \times B^{1}$. If second derived subdivisions of $K$ and $L$ are chosen to agree on $R^{1} \times \partial B^{1} \cup\{0\} \times B^{1}$ and to project simplicially to the subdivision of the triangulation of $\{0\} \times B^{1}$, then there is a fiber preserving PL isotopy of $R^{1} \times B^{1}$, fixed on $R^{1} \times \partial B^{1} \cup\{0\} \times B^{1}$ that takes $N\left(\{0\} \times B^{1}, K^{\prime \prime}\right)$ to $N\left(\{0\} \times B^{1}, L^{\prime \prime}\right)$. (See Figure 1.) We show this is true in a more general but still restricted case (Theorem 12). This is the first result.

The second result (Theorem 11) is that if $X$ is a subcomplex of $Q \times A$, where $Q$ is an arbitrary PL manifold, and $Y$ is a subcomplex of $X$ such that $X$ fiber preserving collapses to $Y$ (definition in $\S 3$ ), then there is a fiber preserving PL ambient isotopy of $Q \times A$ that takes the second derived neighborhood of $X$ onto the second derived neighborhood of $Y$.

Combining the two results gives a still wider class of fiber preserving PL ambient isotopic regular neighborhoods of $Y$.

The drawback of our fibered regular neighborhoods is that the complication goes up with both the number of simplexes in the triangulation of $A$ and the dimension of $A$. See Figure 2 for some examples. This complication results in the inductive spectaculars later in the paper. Keep in mind, however, that no matter what the dimension of $A$ or the complication of its triangulation, the restriction of a fibered regular neighborhood to a single $Q \times\{a\}$ can be any regular neighborhood; it can be flat or lumpy and has no characteristic shape. 


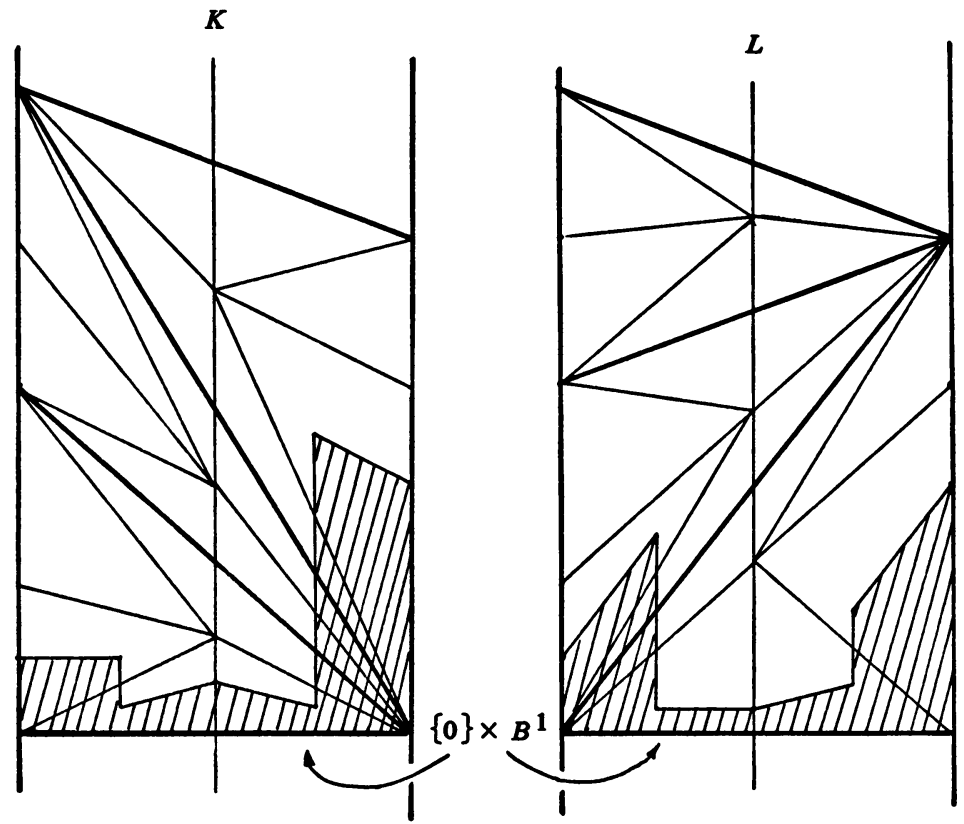

FIGURE 1. The slashed areas denote the second derived neighborhoods of $\{0\} \times B^{1}$ in $R+B^{1}$.
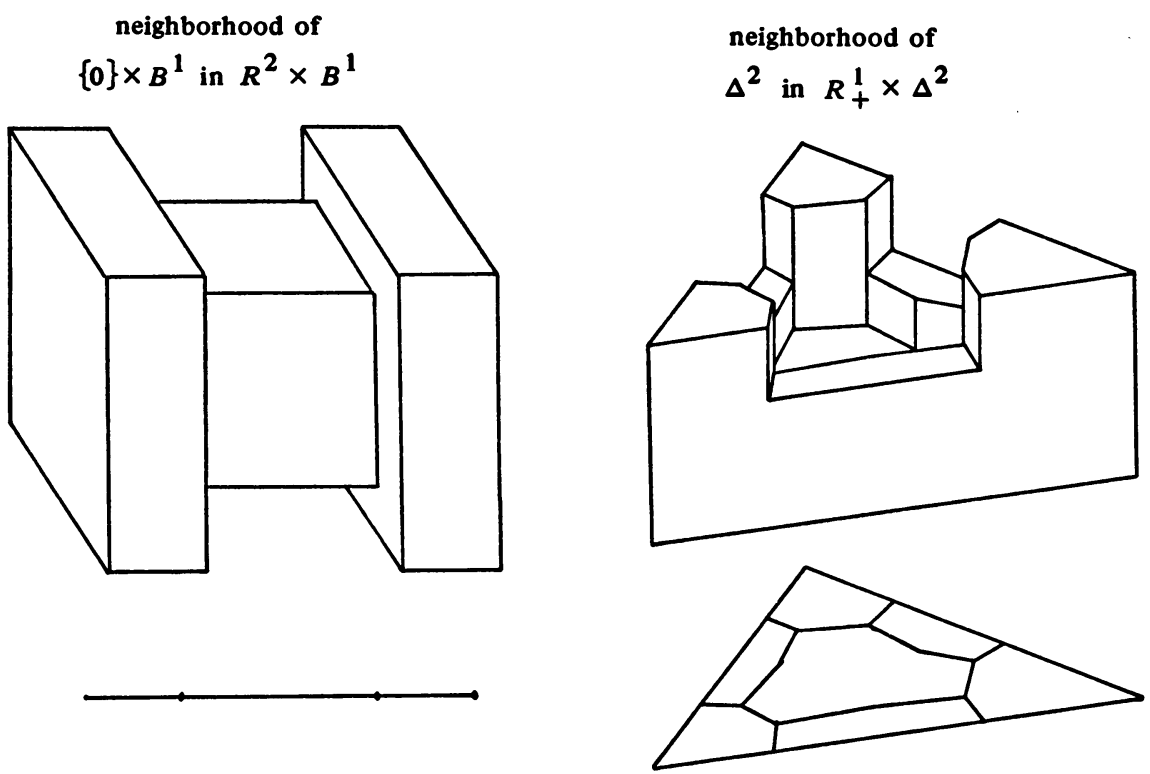

FIGURE 2. Typical fibered neighborhoods. Beneath each neighborhood is the dual subdivision of $A$. 
The following approximation theorem complements the nonfibered equivalence theorem.

Theorem (BRyANT) [Br]. Suppose $f: X \rightarrow$ int $Q$ is a TOP embedding of a compact polyhedron $X$ into a PL manifold $Q$, and suppose $\operatorname{dim} X \leqslant$ $\operatorname{dim} Q-3$. Then given $\epsilon>0$, there is a PL embedding $g: X \rightarrow \operatorname{int} Q$ that $\epsilon$-approximates $f$.

This theorem translates to the statement that the space of codimension 3 PL embeddings of a complex into a PL manifold is dense in the space of TOP embeddings.

We give a quick argument in $\S 1$ that proves a fibered approximation theorem (Theorem 3) using Bryant's approximation theorem and the fibered close equivalence theorem. The argument is really a homotopy result which always follows from local contractibility and density [E \& W].

1. Statement of the equivalence and approximation theorems. Let $Q$ be a PL manifold. Let $X$ be a compact subpolyhedron of $Q$ and $Y$ be a subcomplex of $X$. Let $A$ be a finite complex and $N$ be a subcomplex of $A$. Denote the standard s-simplex by $\Delta^{s}$. A map $h: X \times A \rightarrow Q \times A$ is fiber preserving if it commutes with projection onto the second factor.

TheOREM 1 (Fibered Close EQuivalence). Suppose $\operatorname{dim} X \leqslant Q-4$ and $\operatorname{dim}(X \cap \partial Q) \leqslant \operatorname{dim} Q-5$. Suppose $h: X \rightarrow Q$ is a TOP embedding with $h^{-1}(\partial Q)=X \cap \partial Q$. Let $s$ be a nonnegative integer. Then for $\epsilon>0$ there is $a \delta>0$ such that if $g^{*}: X \rightarrow Q$ is a PL embedding within $\delta$ of $h$, and with $g^{*-1}(\partial Q)=X \cap \partial Q$ and if $g: X \times \Delta^{s} \rightarrow Q \times \Delta^{s}$ is a fiber preserving $P L$ embedding satisfying

(1) $g^{-1}\left(\partial Q \times \Delta^{s}\right)=(X \cap \partial Q) \times \Delta^{s}$,

(2) $g$ is within $\delta$ of $h \times \mathrm{id}_{\Delta^{s}}$,

(3) $g \mid\left(X \times \partial \Delta^{s}\right) \cup\left(Y \times \Delta^{s}\right)=\left(g^{*} \times\right.$ id $\left._{\partial \Delta^{s}}\right) \cup\left(\left(g^{*} \mid Y\right) \times\right.$ id $\left._{\Delta^{s}}\right)$, it follows that there is a fiber preserving PL ambient isotopy $\Theta_{t}$ of $Q \times \Delta^{s}$ with

(4) $\Theta_{1} \circ g=g^{*} \times$ id $_{\Delta^{s}}$,

(5) $\Theta_{t} \mid\left(Q \times \partial \Delta^{s}\right) \cup\left(Y \times \Delta^{s}\right)=$ identity,

(6) $\Theta_{t}$ is within $\epsilon$ of the identity everywhere and is fixed outside the $\epsilon$-neighborhood of $h(X) \times \Delta^{s}$, and

(7) if $Y \supset(X \cap \partial Q)$, then $\Theta_{t} \mid \partial Q \times \Delta^{s}=$ identity.

In the next section we will reduce Theorem 1 to Theorem 4 (below). The proof of Theorem 4 will then occupy the remainder of the paper. 
REMARK 2. For a given $\epsilon>0$, a given integer $s$, and $h: X \rightarrow Q$, the same $\delta>0$ works in Theorem 1 for all TOP embeddings sufficiently near $h$. Thus, in particular, if $H: X \times A \rightarrow Q \times A$ is a fiber preserving embedding and $A$ is any finite complex, there is a single $\delta>0$ which implies the conclusions of Theorem 1 whenever the TOP embedding $h$ is replaced by one of the embeddings $H \mid X \times\{a\}, a \in A$.

Remark 2 together with the work of Bryant gives a quick proof of the next theorem.

Theorem 3 (Fibered APPRoximation). Suppose $\operatorname{dim} X \leqslant \operatorname{dim} Q-4$ and $\operatorname{dim}(X \cap \partial Q) \leqslant \operatorname{dim} Q-5$. Suppose $h: X \times A \rightarrow Q \times A$ is a fiber preserving TOP embedding such that $h \mid(X \times B) \cup(Y \times A)$ is PL. Then for $\epsilon>0$ there is a fiber preserving $P L$ embedding $g: X \times A \rightarrow Q \times A$ such that

(1) $g|(X \times B) \cup(Y \times A)=h|(X \times B) \cup(Y \times A)$,

(2) $g$ is within $\epsilon$ of $h$.

Proof of Theorem 3. By induction on the dimension $k$ of $A-B$. The case $k=0$ is Bryant's approximation theorem. If $k>0$, for $\epsilon>0$, subdivide $A$ to obtain $\tilde{A}$ whose mesh is so small that for each simplex $\sigma \in A, h \mid X \times \sigma$ is within $\delta / 3$ of $(h \mid X \times \hat{\sigma}) \times \mathrm{id}_{\sigma}$ where $\hat{\sigma}$ is the barycenter of $\sigma$ and $\delta$ comes from Remark 2 applied to $\epsilon / 2$. In addition, take $\delta<\epsilon / 2$. Apply Theorem 3 inductively to obtain a $\delta / 3$ approximation $g \mid X \times \widetilde{A}^{(k-1)}$ to $h \mid X \times \widetilde{A}^{(k-1)}$. For each principal simplex $\sigma \in \widetilde{A}$ choose a point $s \in \partial \sigma$. Theorem 1 gives an $\epsilon / 2$-contraction of the $(k-1)$ sphere of PL embeddings $g \mid X \times$ bdy $\sigma$ to the embedding $g \mid X \times\{s\}$. Regard this contraction as a fiber preserving PL embedding $g \mid X \times \sigma$ that extends $g \mid X \times \partial \sigma$. Thus the union over $\sigma \in A$ of the embeddings $g \mid X \times \sigma$ defines a fiber preserving PL embedding $g$ : $X \times A \rightarrow Q \times A$. It is an $\epsilon$-approximation to $h$.

A PL map $f: X \rightarrow Y$ is collapsible if, for each point $y \in Y, f^{-1}(y)$ is a collapsible polyhedron. If $Y$ is a subpolyhedron of $X$ and $f: X \rightarrow Y$ is a collapsible retraction, then $X$ collapses to $Y$ [C, Theorem 8.1]. We define an abstract regular neighborhoood of $X$ to be a compact manifold pair $\left(N^{n}, M^{n-1}\right)$ with $M \subset \partial N$, and an inclusion of pairs $(X, X \cap \partial N) \subset$ (int $N \cup \operatorname{int} M$, int $M)$ such that there is a collapsible retraction $\rho: N \rightarrow X$ whose restriction $\rho \mid M$ is a collapsible retraction of $M$ to $(X \cap \partial N)$. In particular, the second derived neighborhood of a subcomplex in a manifold is an abstract regular neighborhood.

THEOREM $4(n, j, k)$ (FIBERED EQUIVALENCE THEOREM). Let $X$ be a finite complex, and let $Y$ be a subcomplex of $X$. Let $(A, B)$ be a pair of finite complexes. Suppose $\operatorname{dim} X=j$, and $\operatorname{dim} A=k$. Suppose further that $n-j$ $\leqslant 4$. Then for $\epsilon>0$, there is $a \delta>0$ such that if $(N, M, \rho)$ is an abstract 
regular neighborhood of $X$ with $\operatorname{dim} N=n$ and $Y \supset(X \cap \partial N)$, and if $g$ : $X \times A \rightarrow N \times A$ is a fiber preserving embedding with

(1) $g^{-1}(\partial N \times A)=(X \cap \partial N) \times A$,

(2) $g \mid(X \times B) \cup(Y \times A)=$ inclusion, and

(3) $\left(\rho \times \mathrm{id}_{A}\right) \circ g$ is within $\delta$ of the inclusion,

then there is a fiber preserving ambient isotopy $\Theta_{t}$ of $N \times A$ with

(4) $\Theta_{1} \circ g=$ inclusion,

(5) $\Theta_{t} \mid(N \times B) \cup(Y \cup \partial N) \times A=$ identity, and

(6) $\left(\rho \times \mathrm{id}_{A}\right) \circ \Theta_{t}$ is within $\epsilon$ of $\left(\rho \times \mathrm{id}_{A}\right)$.

The feature of abstract regular neighborhoods that we exploit in this paper is contained in the next proposition. It is proved in [C].

PROPOSITION 5. Suppose $(N, M, \rho)$ is an abstract regular neighborhood of $X$. Then for each triangulation $\widetilde{N}$ of $N$ such that the collapsible retraction $\rho$ : $(\widetilde{N}, \widetilde{M}) \rightarrow(\widetilde{X}, \widetilde{X} \cap \partial N)$ is simplicial it follows that $\rho^{-1}\left(D\left(\tau, \widetilde{X}^{\prime \prime}\right)\right)$ is a $(\operatorname{dim} N-\operatorname{dim} \tau)$-ball for each simplex $\tau \in \widetilde{X}^{\prime}$. (See $\$ 3$ for the definition of the dual set $D\left(\tau, \widetilde{X}^{\prime \prime}\right)$.)

It is convenient to state an equivalent form of Theorem 4.

THeOREM $6(n, j, k) . X, Y, A, B$ as in Theorem 4. Suppose $\tilde{X}$ is a triangulation of $X$. Then there is a $\delta>0$ such that if $(N, M, \widetilde{\rho})$ is an abstract regular neighborhood of $X$ with $(Y \supset X \cap \partial N)$ and $\tilde{\rho}^{-1}\left(D\left(\tau, \tilde{X}^{\prime \prime}\right)\right) \quad a$ $\left(n-\operatorname{dim} \tau\right.$ )-ball for each simplex $\tau \in \widetilde{X}^{\prime}$, and if $g: X \times A \rightarrow N \times A$ is a fiber preserving embedding with

(1) $g^{-1}(\partial N \times A)=(X \cap \partial N) \times A$,

(2) $g \mid(X \times B) \cup(Y \times A)=$ inclusion, and

(3) $\left(\tilde{\rho} \times \mathrm{id}_{A}\right) \circ g$ is within $\delta$ of the inclusion,

then there is a fiber preserving ambient isotopy $\Theta_{t}$ of $N \times A$ with

(4) $\Theta_{1} \circ g=$ inclusion,

(5) $\Theta_{t} \mid(N \times B) \cup(Y \cup \partial N) \times A=$ identity, $\mu \in \widetilde{X}$.

(6) $\Theta_{t}\left(\widetilde{\rho}^{-1}(\mu) \times A\right) \subset \tilde{\rho}^{-1}\left(N\left(N\left(\mu, \widetilde{X}^{\prime \prime}\right), \widetilde{X}^{\prime \prime}\right)\right) \times A$ for each simplex

2. Generalities about fibered maps and the reduction of Theorem 1 to Theorem 4. The reduction of Theorem 1 to Theorem 4 has precedent in [B \& S] and $[E]$. First, a few preliminaries.

Proposition 7 (STRAIGHT LINE hOMOTOPIES). Let $W^{w}$ be a topological manifold, $A$ be any metric space, and $S$ be any compact subset of $W \times A$. Then for $\epsilon>0$ there is a $\delta>0$ such that any fiber preserving map of $S$ into $W \times$ $A$ that is within $\delta$ of the inclusion can be covered by a homotopy of the in- 
clusion that itself is within $\epsilon$ of the identity. If $S$ is not compact, both $\epsilon$ and $\delta$ are to be considered functions of $W \times A$ into $(0, \infty)$.

PRoof. If $W=R^{w}$, the straight line homotopy obtained by moving each point in $S$ linearly along the line between it and its image under the map satisfies the proposition with $\delta=\epsilon$.

Extend the proposition to manifolds a coordinate patch at a time. Use the fact that $W$ is the union of $w+1$ collections of disjoint coordinate patches.

Proposition 8(Fibered pl APPRoximation to maps). Let $X, Y, A$ and $B$ be as in $\S 1$. Let $Z$ be a simplicial complex. Choose $\epsilon>0$. If $f: X \times A$ $\rightarrow Z \times A$ is a fiber preserving map whose restriction to $(X \times B) \cup(Y \times A)$ is $P L$, then there is a fiber preserving PL map $g: X \times A \rightarrow Z \times A$ that extends $f \mid(X \times B) \cup(Y \times A)$ and that homotops to $f$ by an $\in$-homotopy.

Proof. If $Z=R^{n}$, choose $\delta>0$ and subdivide $X \times A$ so that the image of each simplex has diameter $<\delta$. Define the restriction of $g$ to the zero skeleton of the subdivided $X \times A$, to agree with the restriction of $f$. Extend linearly.

If $Z$ is an arbitrary complex, PL embed it in $R^{n}$ (for some $n$ ) and let $Q$ be a regular neighborhood with $\epsilon / 2$-PL retraction $\rho: Q \rightarrow Z$. Apply the previous paragraph, with $\delta<\epsilon / 4$ and such that the $2 \delta$-neighborhood of $Z$ lies in $Q$, to define a fiber preserving PL map $g^{\prime}: X \times A \rightarrow Q \times A$. Let $g=\rho \circ g^{\prime}$. Use Proposition 7 to construct a small homotopy of $g^{\prime}$ to $f$ in $Q$. Composition with $\rho$ pushes this homotopy into $Z$.

The following definition is due to Millett. Two subcomplexes $S$ and $T$ of (int $Q) \times A$ are in fibered general position relative to a subcomplex $S_{0}$ of $S$ if

(1) $\operatorname{dim}\left(\left(S-S_{0}\right) \cap T\right) \leqslant \operatorname{dim}\left(S-S_{0}\right)+\operatorname{dim} T-\operatorname{dim}(Q \times A)$,

(2) for each point $a \in A, \operatorname{dim}\left(\left(S-S_{0}\right) \cap T \cap(Q \times\{a\})\right) \leqslant$ $\max \left\{\left(\operatorname{dim}\left(\left(S-S_{0}\right) \cap(Q \times\{a\})\right)+\operatorname{dim}(T \cap(Q \times\{a\}))-\operatorname{dim} Q\right), 0\right\}$.

Warning. The zero in condition 2 has powerful effects on fibered PL theory.

Proposition 9 (fibered general position). See Millett [Mi]. Choose $\epsilon>0$. Let $S$ and $T$ be subcomplexes of (int $Q$ ) $\times A$, where $Q$ is a $P L$ manifold. Let $S_{0}$ be a subcomplex of $S$. Then there is a fiber preserving $P L$ ambient isotopy $\Phi_{t}$ of $Q \times A$ such that

(1) $\Phi_{t} \mid(($ bdy $Q) \times A) \cup S_{0}=$ identity,

(2) $\Phi_{t}$ is within $\epsilon$ of the identity,

(3) $\Phi_{1}(S)$ and $T$ are in fibered general position relative to $S_{0}$.

If $B$ is a subcomplex of $A$, and if $S \cap(Q \times B)$ and $T \cap(Q \times B)$ are 
already in fibered general position relative to $S_{0} \cap(Q \times B)$ then $\Phi_{t}$ fixes $Q \times B$.

Returning to Theorem 1. Since a finite complex is an ANR, there is a neighborhood of $h(X)$ in $Q$ that retracts to $h(X)$. For each sufficiently small $\delta$-neighborhood of $h(X)$ in $Q, N_{\delta}(h(X))$, and any embedding $g^{*}: X \rightarrow Q$ within $\delta$ of $h$, the composition of the retraction with $g^{*} \circ h^{-1}$ is a close map of $N_{\delta}(h(X))$ into $g^{*}(X)$. Since $g^{*}(X)$ is locally contractible, this close map can be converted to a close retraction of $N_{\delta}(h(X))$ to $g^{*}(X)$. Thus by Proposition 7 there is a close homotopy of $N_{\delta}(h(X))$ into $g^{*}(X)$. The track of this homotopy will not generally be in $N_{\delta}(h(X))$, but will be in $N_{\delta^{\prime}}(h(X))$ where $\delta^{\prime}$ is a function of $\delta$ that goes to zero as $\delta$ goes to zero. If $\delta^{\prime}$ is sufficiently small, the process can be repeated to provide a homotopy of $N_{\delta}(h(X))$ into $g^{*}(X)$. This, together with Propositions 8 and 9, are just the components needed for small fibered Stallings-Bing type PL engulfing (cf. [B]) of $g\left(X \times \Delta^{s}\right)$ into $N \times \Delta^{s}$, where $N$ is a small derived neighborhood of $g^{*}(X)$ in $Q$. The engulfing leaves a neighborhood of $g^{*}(X) \times \Delta^{s}$ fixed. This puts the problem in the context of Theorem 4.

3. Fibered PL tools. For the rest of the paper all manifolds and maps are PL. If $X$ is a complex, $X^{\prime}$ denotes a first derived subdivision of $X$. If $Y$ is a subcomplex of $X, N(Y, X)$ denotes the subcomplex of $X$ generated by all simplexes of $X$ that intersect $Y$. We mildly abuse notation and write $N\left(Y, X^{\prime}\right)$ for $N\left(Y \cap X^{\prime}, X^{\prime}\right)$. If a simplex $\tau$ is a face of a simplex $\sigma$, we write $\sigma \geqslant \tau$. If $\sigma \geqslant \tau$ but $\sigma \neq \tau$, we call $\tau$ a proper face of $\sigma$ and write $\sigma>\tau$. A simplex in a complex is principal if it is not a proper face of any simplex in the complex. If $K$ and $L$ are complexes, we denote their join by $K \cdot L$.

If $X$ is a complex and $\tau$ a simplex in $X$, the dual set to $\tau$ in $X^{\prime}$ is

$$
D\left(\tau, X^{\prime}\right)=\bigcap_{\text {vertices; } \nu \text { in } \tau} N\left(\nu, X^{\prime}\right) .
$$

If $\sigma$ and $\tau$ are simplexes in $X$, notice that $D\left(\sigma, X^{\prime}\right) \cap D\left(\tau, X^{\prime}\right)=$ $D\left(\sigma \cdot \tau, X^{\prime}\right)$ if $\sigma \cdot \tau$ is a simplex of $X$ and is empty otherwise. The dual set $D\left(\tau, X^{\prime}\right)$ is a cone with apex at $\hat{\tau}$. If $X$ is a manifold then $D\left(\tau, X^{\prime}\right)$ is a $(\operatorname{dim} X-\operatorname{dim} \tau)$-cell. The frontier of $D\left(\tau, X^{\prime}\right)$ in $X^{\prime}$ equals $\bigcup_{\mu>\tau} D\left(\mu, X^{\prime}\right)$.

Suppose that $M$ is a manifold, that $Q$ is a triangulation of $M \times I^{k}$, that $K$ is a triangulation of $I^{k}$, and that the projection $\pi: Q \rightarrow K$ is simplicial. Suppose $Q^{\prime \prime}$ is any second derived subdivision of $Q$ that projects simplicially to $K^{\prime \prime}$. Then we have

THE FUNDAMENTAL OBSERVATION (SEE FIGURE 3). Let $\tau$ be a simplex of $Q$, and let $\sigma=\pi(\tau)$. Then for each simplex $\sigma^{*}$ in $\partial \sigma, N\left(\hat{\tau}, Q^{\prime \prime}\right) \cap$ 
$N\left(\pi^{-1}\left(\hat{\sigma}^{*}\right) \cap \tau^{\prime}, Q^{\prime \prime}\right)$ is contained in the topological interior of $\pi^{-1}\left(D\left(\hat{\sigma} \hat{\sigma}^{*}, K^{\prime \prime}\right)\right) \cap N\left(\pi^{-1}\left(\hat{\sigma}^{*}\right) \cap \tau^{\prime}, Q^{\prime \prime}\right)$ in $\pi^{-1}\left(D\left(\hat{\sigma} \hat{\sigma}^{*}, K^{\prime \prime}\right)\right)$.
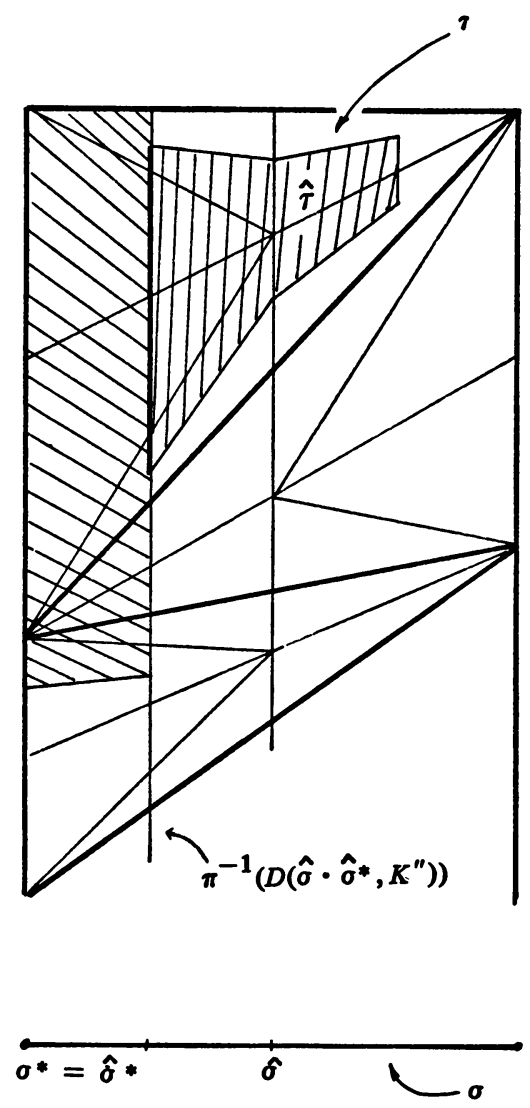

FIGURE 3

Proof.

$N\left(\hat{\tau}, Q^{\prime \prime}\right) \cap N\left(\pi^{-1}\left(\hat{\sigma}^{*}\right) \cap \tau^{\prime}, Q^{\prime \prime}\right)=\bigcup_{\text {vertices; } \nu \in \pi^{-1}\left(\hat{\sigma}^{*}\right)^{\prime} \cap \tau^{\prime}} D\left(\nu \cdot \hat{\tau}, Q^{\prime \prime}\right)$ and

$$
\overline{\pi^{-1}\left(D\left(\hat{\sigma} \hat{\sigma}^{*}, K^{\prime \prime}\right)\right)-N\left(\pi^{-1}\left(\hat{\sigma}^{*}\right) \cap \tau^{\prime}, Q^{\prime \prime}\right)}=\bigcup_{\substack{\boldsymbol{v} \text { ertices } \\ \mu \in \pi^{-1}\left(\hat{\sigma}^{*}\right)^{\prime}-\gamma^{\prime} \\ \omega \in \pi^{-1}(\hat{\sigma})}} D\left(\mu \cdot \omega, Q^{\prime \prime}\right) .
$$

These sets do not intersect since $\mu$ and $\hat{\tau}$ do not span a simplex in $Q^{\prime}$.

LEMMA 10 (DUAL SETS). Let $X$ be a complex. Let $L$ be a triangulation of $X \times I^{k}$ and $K$ be a triangulation of $I^{k}$ such that the projection $\pi: L \rightarrow$ 
$K$ is simplicial. Suppose further that $\pi: L^{\prime \prime} \rightarrow K^{\prime \prime}$ is simplicial. Suppose $\mu$ is a simplex in $L^{\prime}$ and let $\nu=\pi(\mu)$. Then there is a fiber preserving homeomorphism

$$
h_{\mu}: D\left(\nu, K^{\prime \prime}\right) \times\left(D\left(\mu, L^{\prime \prime}\right) \cap \pi^{-1}(\hat{v})\right) \rightarrow D\left(\mu, L^{\prime \prime}\right)
$$

such that

(1) $h_{\mu}$ extends the inclusion of $D\left(\mu, L^{\prime \prime}\right) \cap \pi^{-1}(\hat{\nu})$ in $D\left(\mu, L^{\prime \prime}\right)$,

(2) if $\tau>\mu$ and $\pi(\tau)=\nu$, then $h_{\mu} \mid D\left(\nu, K^{\prime \prime}\right) \times\left(D\left(\tau, L^{\prime \prime}\right) \cap \pi^{-1}(\hat{\nu})\right)=h_{\tau}$,

(3) $h_{\mu}\left(D\left(\nu, K^{\prime \prime}\right) \times\left(D\left(\mu, L^{\prime \prime} \cap \gamma \times I^{\mu}\right) \cap \pi^{-1}(\hat{v})\right)\right)=D\left(\mu, L^{\prime \prime} \cap \gamma \times I^{\mu}\right)$ for each simplex $\gamma \in X$, and

(4) $h_{\mu}\left(D\left(\nu\right.\right.$, bdy $\left.K^{\prime \prime}\right) \times\left(D\left(\mu, L^{\prime \prime} \cap \pi^{-1}(\operatorname{bdy} K) \cap \pi^{-1}(\hat{v})\right)\right)=$ $D\left(\mu, L^{\prime \prime} \cap \pi^{-1}(\right.$ bdy $\left.K)\right)$.

(See Figure 4.)
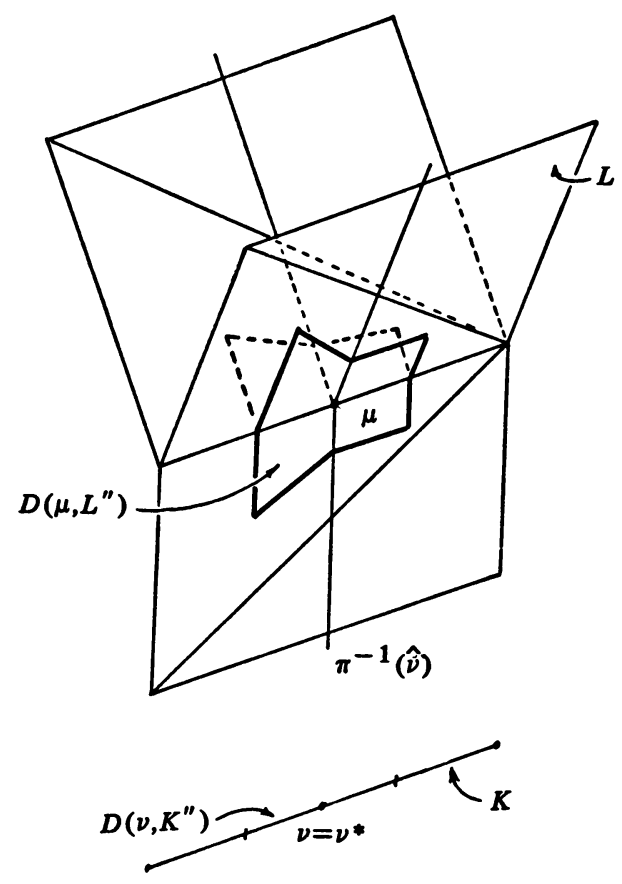

FIGURE 4

Proof. Inductively on the dimension of $K$ we can assume $h_{\mu}$ is already constructed over $\partial K$. By extending this homeomorphism we automatically satisfy (4). By induction on the number of simplexes in $X$, we can assume that $h_{\mu}$ is defined over all but one principal simplex of $X$. Thus we can assume that $X$ is a single simplex and that $h_{\mu}$ is already defined on its boundary. This takes care of (3). We will extend this homeomorphism. 
Proceed by double induction, first on decreasing dimension of $\nu$, and then on decreasing discussion of $\mu$. The induction starts trivially since the identity map satisfies the lemma if $\operatorname{dim} \nu=\operatorname{dim} K$.

For an arbitrary simplex $\mu$, inductively by (2), the homeomorphism $h_{\tau}, \tau>$ $\mu$ with $\pi(\tau)=\nu$, and the homeomorphism over $\partial K$ fit together to give a fiber preserving homeomorphism

$$
\begin{aligned}
h_{\partial}: D\left(\nu, K^{\prime \prime}\right) & \times\left(\partial D\left(\mu, L^{\prime \prime}\right) \cap \pi^{-1}(\nu)\right) \\
& \rightarrow \bigcup_{\tau>\mu ; \pi(\tau)=\nu} D\left(\tau, L^{\prime \prime}\right) \cup\left(D\left(\mu, L^{\prime \prime}\right) \cap\left(\partial X \times I^{k}\right)\right) .
\end{aligned}
$$

Also by induction, for each simplex $\lambda \in K^{\prime}$ with $\lambda>\nu$ and $\operatorname{dim} \lambda=$ $\operatorname{dim} v+1$, there is a fiber preserving homeomorphism

$$
\left.\mu_{\lambda}: D\left(\lambda, K^{\prime \prime}\right) \times\left(D\left(\mu, L^{\prime \prime}\right) \cap \pi^{-1} \hat{\lambda}\right)\right) \rightarrow \bigcup_{\tau>\mu ; \pi(\tau)=\lambda} D\left(\tau, L^{\prime \prime}\right)
$$

Notice that $D\left(\mu, \pi^{-1}(\lambda)^{\prime}\right)$ is a ball that contains $D\left(\mu, \pi^{-1}(\nu)^{\prime}\right)$ as a top dimensional ball in its boundary. Because

$$
\begin{array}{r}
\bigcup_{\tau>\mu ; \pi(\tau)=\nu} D\left(\tau, \pi^{-1}(\lambda)^{\prime}\right) \cup D\left(\mu, \pi^{-1}(\lambda)^{\prime} \cap\left(\partial X \times I^{k}\right)\right) \\
=h_{\partial}\left(\partial D \mu, \pi^{-1}(\nu)^{\prime} \times \hat{\nu} \cdot \hat{\lambda}\right),
\end{array}
$$

the left-hand side is a collar for $D\left(\mu, \pi^{-1}(\nu)^{\prime}\right)$ in $\partial D\left(\mu, \pi^{-1}(\lambda)^{\prime}\right)$. We also have

$$
\begin{aligned}
\text { closure } & \left\{\partial D\left(\mu, \pi^{-1}(\lambda)^{\prime}\right)\right. \\
& \left.-\left[D\left(\mu, \pi^{-1}(\lambda)^{\prime} \cap\left(\partial X \times I^{k}\right)\right) \cup \bigcup_{\tau>\mu ; \pi(\tau)=\nu} D\left(\tau, \pi^{-1}(\lambda)^{\prime}\right)\right]\right\} \\
= & D\left(\mu, L^{\prime \prime}\right) \cap \pi^{-1}(\hat{\lambda}) .
\end{aligned}
$$

Thus, the right-hand side is a ball homeomorphic to $D\left(\mu, L^{\prime \prime}\right) \cap \pi^{-1}(\hat{v})$. Use this homeomorphism to extend $h_{\partial}$ by

$$
\left.h^{\lambda, \mathrm{I}}: \hat{\lambda}\right] \times\left(D\left(\mu, L^{\prime \prime}\right) \cap \pi^{-1}(\hat{\nu})\right) \rightarrow D\left(\mu, L^{\prime \prime}\right) \cap \pi^{-1} \hat{(\lambda)}
$$

and this, using $\mu_{\lambda}$, to obtain fiber preserving

$$
h^{\lambda, \mathrm{II}}: D\left(\lambda, K^{\prime \prime}\right) \times\left(D\left(\mu, L^{\prime \prime}\right) \cap \pi^{-1}(\hat{\nu})\right) \rightarrow D\left(\mu, L^{\prime \prime}\right) \cap \pi^{-1}\left(D\left(\lambda, K^{\prime \prime}\right)\right) \text {. }
$$

Finally, Alexander's trick can be used to fiber preserving isotop the homeomorphisms $h^{\lambda, \text { II }}$ relative $h_{\partial}$ to

$$
h^{\lambda, \mathrm{III}}: D\left(\lambda, K^{\prime \prime}\right) \times\left(D\left(\mu, L^{\prime \prime}\right) \cap \pi^{-1}(\hat{\nu})\right) \rightarrow D\left(\mu, L^{\prime \prime}\right) \cap \pi^{-1}\left(D\left(\lambda, K^{\prime \prime}\right)\right)
$$

which have the property that for each pair of simplexes $\lambda$ and $\bar{\lambda}$ contained in a simplex of $K^{\prime}$ with $\lambda>\nu, \bar{\lambda}>\nu$ and $\operatorname{dim} \lambda=\operatorname{dim} \bar{\lambda}=\operatorname{dim} \nu+1$ 


$$
\begin{aligned}
h^{\lambda, \mathrm{III}} \mid D\left(\lambda \bar{\lambda}, K^{\prime \prime}\right) & \times\left(D\left(\mu, L^{\prime \prime}\right) \cap \pi^{-1}(\hat{\nu})\right) \\
= & h^{\bar{\lambda}, \mathrm{III}} \mid D\left(\lambda \bar{\lambda}, K^{\prime \prime}\right) \times\left(D\left(\mu, L^{\prime \prime}\right) \cap \pi^{-1}(\hat{\nu})\right) .
\end{aligned}
$$

Define

$$
\begin{aligned}
h_{\mu} l & : D\left(\nu, K^{\prime \prime}\right) \times\left(\operatorname{frontier}\left(D\left(\mu, L^{\prime \prime}\right) \cap \pi^{-1}(\hat{v})\right) \text { in } \pi^{-1}(\hat{\nu})\right) \\
& \cup \bigcup_{\lambda>\nu ; \operatorname{dim} \lambda=\operatorname{dim} \nu+1} D\left(\lambda, K^{\prime \prime}\right) \times\left(D\left(\mu, L^{\prime \prime}\right) \cap \pi^{-1}(\hat{\nu})\right) \\
& \rightarrow \bigcup_{\tau>\mu ; \pi(\mu)=\nu} D\left(\tau, L^{\prime \prime}\right) \cup \bigcup_{\lambda>\nu ; \operatorname{dim} \lambda=\operatorname{dim} \nu+1}\left(D\left(\mu, L^{\prime \prime}\right)\right) \cap \pi^{-1}\left(D\left(\lambda, K^{\prime \prime}\right)\right)
\end{aligned}
$$

by $h_{\mu} \mid=h_{\partial} \cup \bigcup_{\lambda>\nu ; \operatorname{dim} \lambda=\operatorname{dim} \nu+1} h^{\lambda, \text { III }}$. This extends by coning to yield $h_{\mu}$. It is easy to check that (1) and (2) hold for $h_{\mu}$.

If $\tau$ is a principal simplex of $X$, and if $\tau_{f}$ is a face of $\tau$ that lies in no other simplex of $X$ we say $\tau_{f}$ is a free face, and that $X$ collapses to $X-$ int $\tau$-int $\tau_{f}$ through $\tau_{f}$, or that there is an elementary collapse of $X$ to $X-$ int $\tau$-int $\tau_{f}$. Notice $X$ collapses to $X-$ int $\tau-$ int $\tau_{f}$ implies $X-$ int $\tau-$ int $\tau_{f}$ is a strong deformation retract of $X$. (The retraction can be made PL but not simplicial.) If $Z$ is a subcomplex of $X$, define the image of $Z$ under the collapse to be the image of $Z$ under any such deformation retraction. It is a subcomplex of $X$-int $\tau-$ int $\tau_{f}$ and does not depend on the retraction chosen. Define the track of $Z$ under the collapse similarly. The composition of a sequence of elementary collapses is called simply a collapse. The image and track of a subcomplex $Z$ of $X$ are, in this case, the image and track of $Z$ under the composition of the.corresponding deformation retractions.

Some more definitions: Suppose $M$ is a manifold, $Q$ is a triangulation of $M \times I^{k}, K$ is a triangulation of $I^{k}$, and $\pi: Q \rightarrow K$ is simplicial. An elementary collapse of $X \subset Q$ to $X-$ int $\tau-$ int $\tau_{f}$ is fiber preserving if $\pi(\tau)=\pi\left(\tau_{f}\right)$. The composition of fiber preserving elementary collapse is a fibered collapse. Notice that although the retractions associated with fiber preserving collapses are not themselves fiber preserving, the image and track of a fiber preserving collapse still make sense. If $X$ collapses to $Y$ write image $_{X \backslash Y} Z$ and $\operatorname{track}_{X \backslash Y} Z$ for the image and track.

THEOREM 11 (ISOTOPY FOLlOWING COLLAPSE). Add to the above notation that $\pi: Q^{\prime \prime} \rightarrow K^{\prime \prime}$ is simplicial. Suppose $X$ is a subcomplex of $Q$ and that $X$ fiber preserving collapses to a subcomplex $Y$. Suppose, too, that $X-$ $Y \subset$ int $Q$. Then there is a fiber preserving ambient isotopy $\Omega_{t}$ of $Q$, fixed outside $N\left(N\left(\right.\right.$ vertices in $\left.\left.X^{\prime}-Y^{\prime}, Q^{\prime \prime}\right), Q^{\prime \prime \prime}\right)$ such that

(1) $\Omega_{1} N\left(X, Q^{\prime \prime}\right)=N\left(Y, Q^{\prime \prime}\right)$,

(2) each point $p \in N\left(X, Q^{\prime \prime}\right)$ is contained in $N\left(N\left(\hat{\delta}, Q^{\prime \prime}\right), Q^{\prime \prime \prime}\right)$ for a unique 
highest dimensional simplex $\delta \in X$, and $\Omega_{1}(p) \in N\left(N\left(\hat{\gamma}, Q^{\prime \prime}\right), N\left(X, Q^{\prime \prime}\right)^{\prime}\right)$ for some simplex $\gamma \in$ image $_{X \backslash Y} \delta$, and

(3) each point $p \in N\left(N\left(X, Q^{\prime \prime}\right), Q^{\prime \prime \prime}\right)$ is contained in $N\left(N\left(\hat{\delta}, Q^{\prime \prime}\right), Q^{\prime \prime \prime}\right)$ for a unique highest dimensional simplex $\delta \in X$, and $\Omega_{[0,1]}(p) \in$ $\cup_{\omega} N\left(N\left(\hat{\omega}, Q^{\prime \prime}\right), Q^{\prime \prime \prime}\right)$ where $\omega$ is a simplex in $\operatorname{track}_{X \backslash Y} \delta$.

Proof. It is sufficient to suppose that $X-Y$ is precisely a principal simplex $\tau$ and a free face $\tau_{f}$, with $\pi(\tau)=\pi\left(\tau_{f}\right)=\sigma$. Use the dual cell lemma to construct a fiber preserving homeomorphism $h: N\left(\hat{\sigma}, K^{\prime \prime}\right) \times \pi^{-1}(\hat{\sigma}) \longrightarrow$ $N\left(\pi^{-1}(\hat{\sigma}), Q^{\prime \prime}\right)$ such that $h\left(N\left(\hat{\sigma}, K^{\prime \prime}\right) \times N\left(\hat{\mu}, \pi^{-1}(\hat{\sigma})^{\prime \prime}\right)\right)=N\left(\hat{\mu}, Q^{\prime \prime}\right)$ for all simplexes $\mu \in Q$ with $\pi(\mu)=\sigma$. Since $N\left(X \cap \pi^{-1}(\hat{\sigma}), \pi^{-1}(\hat{\sigma})^{\prime \prime}=\right.$ $N\left(Y \cap \pi^{-1}(\hat{\sigma})^{\prime \prime}\right) \cup N\left(\hat{\tau} \cdot \hat{\tau}_{f}, \pi^{-1}(\hat{\sigma})^{\prime \prime}\right)$ where $N\left(\hat{\tau} \cdot \hat{\tau}_{f}, \pi^{-1}(\hat{\sigma})^{\prime \prime}\right)$ is a top-dimensional ball in $\operatorname{int}\left(\pi^{-1}(\hat{\sigma})\right)$ that intersects $N\left(Y, \pi^{-1}(\sigma)^{\prime \prime}\right)$ in a top-dimensional ball in its boundary, it follows that for each regular neighborhood $Z$ of $N\left(\hat{\tau} \cdot \hat{\tau}_{f}, \pi^{-1}(\hat{\sigma})^{\prime \prime}\right)$ in $\pi^{-1}(\hat{\sigma})$ there is an ambient isotopy of $\pi^{-1}(\hat{\sigma})$, fixed outside $Z$ that takes $N\left(X, \pi^{-1}(\hat{\sigma})^{\prime \prime}\right)$ onto $N\left(Y, \pi^{-1}(\hat{\sigma})^{\prime \prime}\right)$. Use $h$ to extend this ambient isotopy to a fiber preserving ambient isotopy of $N\left(\pi^{-1}(\hat{\sigma}), Q^{\prime \prime}\right)$. Notice that the extension takes $N\left(X, Q^{\prime \prime}\right) \cap \pi^{-1}$ (int $N\left(\hat{\sigma}, K^{\prime \prime}\right)$ ) onto $N\left(Y, Q^{\prime \prime}\right)$ $\cap \pi^{-1}$ (int $N\left(\sigma, K^{\prime \prime}\right)$ ).

Construct a small fiber preserving collar of $N\left(\pi^{-1}(\hat{\sigma}), Q^{\prime \prime}\right)$ in $Q$, and use this collar in the usual way to extend the fiber preserving isotopy we just constructed to a fiber preserving ambient isotopy $\Omega_{t}$ of $Q$ itself that is fixed outside the collar.

If we choose $Z$ sufficiently small, we find by the fundamental observation that $\overline{N\left(X, Q^{\prime \prime}\right)-N\left(\pi^{-1}(\hat{\sigma}), Q^{\prime \prime}\right)}\left(=\overline{N\left(Y, Q^{\prime \prime}\right)-N\left(\pi^{-1}(\hat{\sigma}), Q^{\prime \prime}\right)}\right)$ remains setwise invariant. Thus $\Omega_{1}\left(N\left(X, Q^{\prime \prime}\right)\right)=N\left(Y, Q^{\prime \prime}\right)$. Small choice of $Z$ and the collar also insures that $\Omega_{t}$ is the identity outside $N\left(N\left(\tau \cdot \tau_{f}, Q^{\prime \prime}\right), Q^{\prime \prime \prime}\right)$. Thus $\Omega_{t}$ satisfies (2) and (3).

Definition of $P_{K}$. Let $J=[-4, k+1]$. If $K$ is a triangulation of $I^{k}$, we define the set $P_{K}$ in $J \times K$ to be

$$
\bigcup_{\sigma \in K}\{k-\operatorname{dim} \sigma\} \times N\left(\hat{\sigma}, K^{\prime \prime}\right)
$$

$\cup \bigcup_{\sigma, \tau \in K}[k-\operatorname{dim} \sigma, k-\operatorname{dim} \tau] \times\left(N\left(\hat{\sigma}, K^{\prime \prime}\right) \cap N\left(\hat{\tau}, K^{\prime \prime}\right)\right)$.

Then $J \times K-P_{K}$ has two components whose closures we call $P_{K}^{+}$and $P_{K}^{-}$. Suppose $L$ is a triangulation of $X \times J \times I^{k}$ that projects simplicially to $K$, and suppose that $X \times\{j\} \times I^{k}$ is the underlying space of a subcomplex of $L$ for integers $-4 \leqslant j \leqslant k+1$. We then have the following theorem.

THEOREM 12 (SPECIAL UNIQUENESS OF REGULAR NEIGHBORHOODS). SUP- 
pose $W$ is a subcomplex of $L$ contained in $X \times[-4,-1] \times I^{k}$ and containing $X \times[-4,-3] \times I^{k}$ such that for each simplex $\tau \in K$, some subdivision of $L \cap$ $(X \times[-4,-1] \times\{\hat{\tau}\})$ collapses to a subdivision of $W \cap(X \times[-4$, $-1] \times\{\hat{\tau}\})$. If $X$ is not a manifold, suppose that under the collapse $\gamma \times J \times\{\hat{\tau}\}$ remains invariant, each simplex $\gamma \in X$. Then there is a fiber preserving isotopy $\Lambda_{t}$ of $X \times J \times I^{k}$ such that

(1) $\Lambda_{1}\left(X \times P_{K}^{-}\right)=N\left(W, L^{\prime \prime}\right)$,

(2) $\Lambda_{t}=$ identity outside an arbitrary neighborhood of

$$
\overline{X \times P_{\bar{K}}^{-}-N\left(W, L^{\prime \prime}\right)} \text {, }
$$

(3) the diameter of $\pi_{X \times I}{ }^{k} \circ \Lambda_{[0,1]}(p)$ is small if the projected tracks of points under the collapses, and the mesh of $K$, are small, and

(4) $\gamma \times J \times I^{k}$ is invariant under $\Lambda_{t}$ if it is under the collapses.

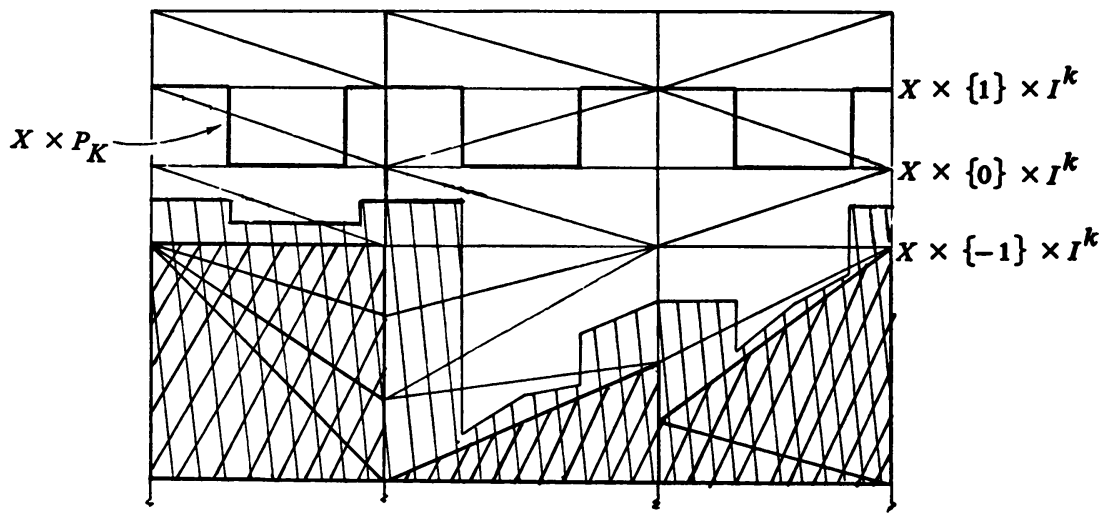

FIGURE 5. Special uniqueness in the case $X=$ point, $k=1$. The cross hatched subcomplex is $W$. The slashed region is $\Lambda_{1}\left(X \times P_{K}^{-}\right)$.

Proof (See Figure 5). Let

$$
N_{\tau}=N\left(W, L^{\prime \prime} \cap\left(X \times J \times N\left(\hat{\tau}, K^{\prime \prime}\right)\right)\right)
$$

and let

$$
X \times P_{\tau}^{-}=X \times[-4, k-\operatorname{dim} \tau] \times N\left(\hat{\tau}, K^{\prime \prime}\right) .
$$

By the dual cell lemma there is a fiber preserving homeomorphism

$$
\begin{aligned}
& h_{\tau}: N\left(\hat{\tau}, K^{\prime \prime}\right) \times(X \times[-4, k-\operatorname{dim} \tau] \times\{\hat{\tau}\}) \\
& \rightarrow N\left(\pi^{-1}(\hat{\tau}), L^{\prime \prime} \cap\left(X \times[-4, k-\operatorname{dim} \tau] \times I^{k}\right)\right)
\end{aligned}
$$

such that

$$
\begin{aligned}
& h_{\tau}\left(N\left(\hat{\tau}, K^{\prime \prime}\right) \times N\left(\hat{\mu}, L^{\prime \prime} \cap(\gamma \times[-4, k-\operatorname{dim} \tau] \times\{\hat{\tau}\})\right)\right) \\
& =N\left(\hat{\mu}, L^{\prime \prime} \cap\left(\gamma \times[-4, k-\operatorname{dim} \tau] \times I^{k}\right)\right)
\end{aligned}
$$


for simplexes $\mu \in L$ with $\pi(\mu)=\tau$ and $\gamma \in X$. Thus, in particular,

$$
\begin{aligned}
\left.\left.h_{\tau}\left(N\left(\hat{\tau}, K^{\prime \prime}\right) \times\left(\overline{\left(X \times P_{K}^{-}-N\left(W, L^{\prime \prime}\right.\right.}\right) \cap \pi^{-1}(\hat{\tau})\right)\right)\right) \\
=\overline{X \times P_{\tau}^{-}-N_{\tau} .}
\end{aligned}
$$

By nonfibered regular neighborhood theory, there is an isotopy of $\pi^{-1}(\hat{\tau})$, fixed outside a neighborhood of $\overline{X \times P_{\tau}^{-}-N_{\tau}} \cap \pi^{-1}(\hat{\tau})$, that takes $\left(X \times P_{\tau}^{-}\right) \cap$ $\pi^{-1}(\hat{\tau})$ onto $N_{\tau} \cap \pi^{-1}(\hat{\tau})$. The projection onto $X \times I^{k}$ of the tracks of this isotopy are small if the projected tracks of the collapse of $L \cap(X \times[-4,-1]$ $\times\{\hat{\tau}\})$ are small. Similarly, the isotopy preserves whatever $\gamma \times J \times\{\hat{\tau}\}$ the collapse does.

Use $h_{\tau}$ to extend this isotopy to a fiber preserving isotopy of $\pi^{-1}\left(N\left(\hat{\tau}, K^{\prime \prime}\right)\right)$. Extend this isotopy to a fiber preserving isotopy $\Lambda_{t}^{\tau}$ of $X \times$ $J \times I^{k}$ by tailing it off to the identity in a small collar of $\pi^{-1}\left(N\left(\hat{\tau}, K^{\prime \prime}\right)\right)$ in $X \times J \times I^{k}$. Then $\Lambda_{t}^{\tau}$ and $\Lambda_{t}^{\bar{\tau}}$, for simplexes $\tau$ and $\bar{\tau}$ of the same dimension, have disjoint support. Let $\Lambda_{t}^{j}$ be the composition of all $\Lambda_{t}^{\tau}$ where $\operatorname{dim} \tau=j$. Let $\Lambda_{t}$ be the isotopy obtained by running through the $\Lambda_{t}^{j}$ in order of decreasing $j$. Sufficiently small choices for the support of the $\Lambda_{t}^{\tau}$ insure, by the fundamental observation, that $\Lambda_{t}^{j}$ fixes $\bigcup_{\operatorname{dim} \mu>j} N_{\mu}$ and leaves $\bigcup_{\operatorname{dim} \mu<j} X \times P_{\mu}^{-}$invariant. Thus, $\Lambda_{t}$ satisfies conditions (1) and (2). Conditions (3) and (4) are satisfied by the individual $\Lambda_{t}^{\tau}$ and are therefore satisfied by the composition.

We will need a corollary of the following simple isotopy extension lemma.

Lemma 13. Let $X$ be a complex and $Y$ be a subcomplex of $X$. Then for $\delta>0$ if $\phi_{t}$ is a fiber preserving ambient isotopy of $(Y \times J) \times I^{k}$ with

(1) $\phi_{t}\left(\tau \times J \times I^{k}\right)=\tau \times J \times I^{k}$, each simplex $\tau \in Y$ and each $t \in[0,1]$,

(2) $\phi_{t} \mid Y \times \partial J \times I^{k}=$ identity,

it follows that there is a fiber preserving ambient isotopy $\Phi_{t}$ of $(X \times J) \times I^{k}$ such that

(3) $\Phi_{t} \mid Y \times J \times I^{k}=\phi_{t}$,

(4) $\Phi_{t}$ is the identity outside the $\delta$-neighborhood of $Y \times J \times I^{k}$ in $X \times J \times I^{k}$,

(5) $\Phi_{t} \mid Y \times \partial J \times I^{k}=$ identity, and

(6) for each point $x$ in the $\delta$-neighborhood, there is a point $y \in Y \times$ $J \times I^{k}$ such that $\Phi_{[0,1]}(x)$ is contained in the $\delta$-neighborhood of $\phi_{[0,1]}(y)$.

In addition, if $\phi_{t} \mid Y \times J \times\{s\}$ is the identity, $t \in[0,1]$, then so is $\Phi_{t} \mid X \times J \times\{s\}$. Finally, all mention of the $J$ factor can be omitted without affecting the truth of the lemma.

Proof. Induction on the number of simplexes in $X$ not in $Y$ reduces 
the lemma to the case $X$ a simplex and $Y$ its boundary. Let $C$ be a small fiber preserving collar of $Y \times J \times I^{k}$ in $X \times J \times I^{k}$. This means there is a fiber preserving homeomorphism $h: Y \times J \times I^{k} \times[0,1] \rightarrow C$ such that $h\left(Y \times J \times I^{k} \times\{0\}\right)=Y \times J \times I^{k}$. Define, for $q \in X \times J \times I^{k}$,

$$
\Phi_{t}(q)= \begin{cases}h\left(\varphi_{t-w}(y, j, s)\right) & \text { if } q=h(y, j, s, w) \text { and if } t-w \geqslant .0 \\ \text { identity } & \text { otherwise. }\end{cases}
$$

To satisfy (4) the collar has to be at least $\delta$-small. Choose such a collar and restrict it to the subcollar $h\left(Y \times J \times I^{k} \times\left[0, \delta^{\prime}\right]\right)$. If $\delta^{\prime}$ is small enough, and the collar $C$ is replaced in the above construction by $h\left(Y \times J \times I^{k} \times\left[0, \delta^{\prime}\right]\right)$, then uniform continuity implies (6). The other conclusions of the lemma are immediate.

We quote the following theorem of Hudson. See [H, Theorem 2] for a proof of the case $Y$ a manifold. The general case follows from Lickorish's cone unknotting theorem.

THEOREM 14 (COVERING $k$-ISOTOPY). Suppose $Y$ is a subcomplex of a manifold $M$, and $\operatorname{dim} Y \leqslant \operatorname{dim} M-3$. Then if $g: Y \times I^{k} \rightarrow M \times I^{k}$ is a fiber preserving embedding for which $g^{-1}\left(\partial M \times I^{k}\right)=(Y \cap \partial M) \times I^{k}$, there is a fiber preserving homeomorphism $G: M \times I^{k} \rightarrow M \times I^{k}$ such that $G \mid Y \times I^{k}$ $=g$.

CoROllary 15. Let $Y$ be a subcomplex of a manifold $M$, with $\operatorname{dim} Y$ $\leqslant \operatorname{dim} M-3$ and $\operatorname{dim}(Y \cap \partial M) \leqslant \operatorname{dim} M-4$. Let $g: Y \times J \times I^{k} \rightarrow M \times$ $J \times I^{k}$ be a fiber preserving embedding with $g\left(Y \times J \times I^{k}\right) \cap\left(\partial M \times J \times I^{k}\right)=$ $g\left((Y \cap \partial M) \times J \times I^{k}\right)$. Then for $\epsilon>0$ if $\phi_{t}$ is a fiber preserving isotopy of $(Y \cup \partial M) \times J \times I^{k}$ with

(1) $\phi_{t}\left(\tau \times J \times I^{k}\right)=\tau \times J \times I^{k}, t \in[0,1]$, each simplex $\tau \in(Y \cup \partial M)$,

(2) $\phi_{t} \mid Y \times \partial J \times I^{k}=$ identity,

it follows that there is a fiber preserving ambient isotopy $\Phi_{t}$ of $M \times J \times I^{k}$ such that

(3) $\Phi_{t} \circ g=g \circ \phi_{t}$,

(4) $\Phi_{t}$ is the identity outside the $\epsilon$-neighborhood of $g((Y \cup \partial M) \times J \times$ $\left.I^{k}\right)$ in $M \times J \times I^{k}$

(5) $\Phi_{t} \mid M \times \partial J \times I^{k}=$ identity, and

(6) for each point $x$ in the $\epsilon$-neighborhood of $g\left((Y \cup \partial M) \times J \times I^{k}\right)$ there is a point $y \in(Y \cup \partial M) \times J \times I^{k}$ such that $\Phi_{[0,1]}(x)$ is contained in the $\epsilon$-neighborhood of $g\left(\phi_{[0,1]}(\gamma)\right)$.

Moreover, if $\phi_{t} \mid(Y \cup \partial M) \times J \times\{s\}$ is the identity, $t \in[0,1]$, then $\Phi_{t} \mid M \times J$ $\times\{s\}$ is the identity, too. Finally, all mention of the $J$ factor can be deleted and the corollary still holds. 
Proof. Apply Theorem 14 to the $k$-isotopy $g$ to obtain a covering homeomorphism $G$ of $M \times J \times I^{k}$ to itself. Use Lemma 13 on the isotopy $\phi_{t}$ of $Y \times J \times I^{k}$ being careful to choose $\delta$ in Lemma 13 so small that the image under $G$ of $\delta$ balls in $M \times J \times I^{k}$ have diameter less than $\epsilon$. Then $G \circ \Phi_{t}$ (Lemma 13) $=\Phi_{t}$ (Corollary 15) is the required isotopy.

4. Proof of the fibered equivalence theorem. The idea of the proof is illustrated in Figures 6-7. We show the nonfibered case, but the same outline is followed in general. Of course, there is an additional induction on the dimension of the indexing complex $A$. One might hope this would be all. In fact, the complicated form of the fibered regular neighborhoods (see Introduction) leads us to incorporate first an addendum to Theorem 4, and then a frilly slicing lemma into the inductive scheme.

The grand induction starts with Theorem $4(n-1)$, Theorem $4(n, k-1)$, and Theorem $4(n, j-1, k)$. (We omit indices to indicate that they may take arbitrary values, although $j$ is always subject to the constraint $n-j \geqslant 4$.) It ends by showing the truth of Theorem $6(n)$ in the case $A=I^{k}, B=\partial I^{k}, \operatorname{dim} X=j$, and $Y$ contains the $(j-1)$-skeleton of $X$. This easily implies Theorem $4(n)$.

Corollary $16(n, j, k)$ (ADdendum to Theorem 4). For $\epsilon>0$, there is $a \delta>0$ such that if $g:(X \times[0,1]) \times A \rightarrow(N \times[0,1]) \times A$ is fiber preserving in the $A$ factor, if

(1) $g \mid(Y \times[0,1] \times A) \cup(X \times\{0,1\} \times A) \cup(X \times[0,1] \times B)=$ identity, and if

(2) $\left(\rho \times \mathrm{id}_{A}\right) \circ \pi_{N \times A} \circ g$ is within $\delta$ of $\left(\rho \times \mathrm{id}_{A}\right) \circ \pi_{N \times A} \circ$ inclusion, then there is a fiber preserving ambient isotopy $\Psi_{t}$ of $(N \times[0,1]) \times A$ with

(3) $\Psi_{1} \circ g=$ identity,

(4) $\Psi_{t} \mid(Y \times[0,1] \times A) \cup(N \times\{0,1\} \times A) \cup(N \times[0,1] \times B)=$ identity, and

(5) $\left(\rho \times \mathrm{id}_{A}\right) \circ \pi_{N \times A} \circ \Psi_{t}$ is within $\epsilon$ of $\left(\rho \times \mathrm{id}_{A}\right) \circ \pi_{N \times A}$. If $Y \supset(X \cap \partial N)$, then $\Psi_{t} \mid \partial N \times[0,1] \times A=$ identity.

Proof. For an arbitrary positive even integer $w$ we can choose $0<$ $\alpha_{1}<\alpha_{2}<\cdots<\alpha_{w}<1 / 2$ such that

$$
\begin{aligned}
& g\left(X \times\left[\alpha_{2 j}, 1-\alpha_{2 j}\right] \times I^{k}\right) \subset N \times\left[\alpha_{2 j-1}, 1-\alpha_{2 j-1}\right] \times I^{k} \text { and } \\
& g\left(X \times\left[\alpha_{2 j}, 1-\alpha_{2 j}\right] \times I^{k}\right) \cap\left(N \times\left[\alpha_{2 j+1}, 1-\alpha_{2 j+1}\right] \times I^{k}\right) \\
& =g\left(X \times[0,1] \times I^{k}\right) \cap\left(N \times\left[\alpha_{2 j+1}, 1-\alpha_{2 j+1}\right] \times I^{k}\right)
\end{aligned}
$$

all $0<j<w / 2$. Let $\omega_{t}$ be an isotopy of $[0,1]$ that is fixed on $\{0,1\}$ and such that $\omega_{1}\left(\left[\alpha_{j}, 1-\alpha_{j}\right]\right)=[j / 2 w, 1-j / 2 w], 0<j<w$. Let $\Omega_{t}=\mathrm{id} \times$ 


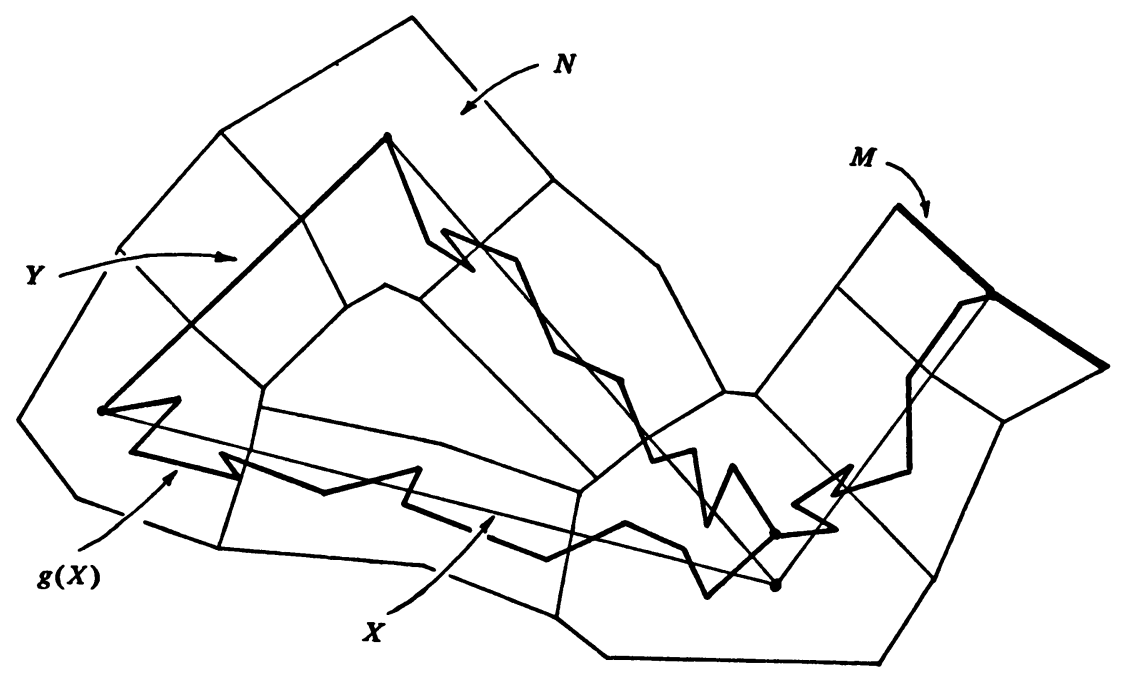

FIGURE 6. The hypothesis of Theorem $4, k=0$. Dimension restrictions are ignored.

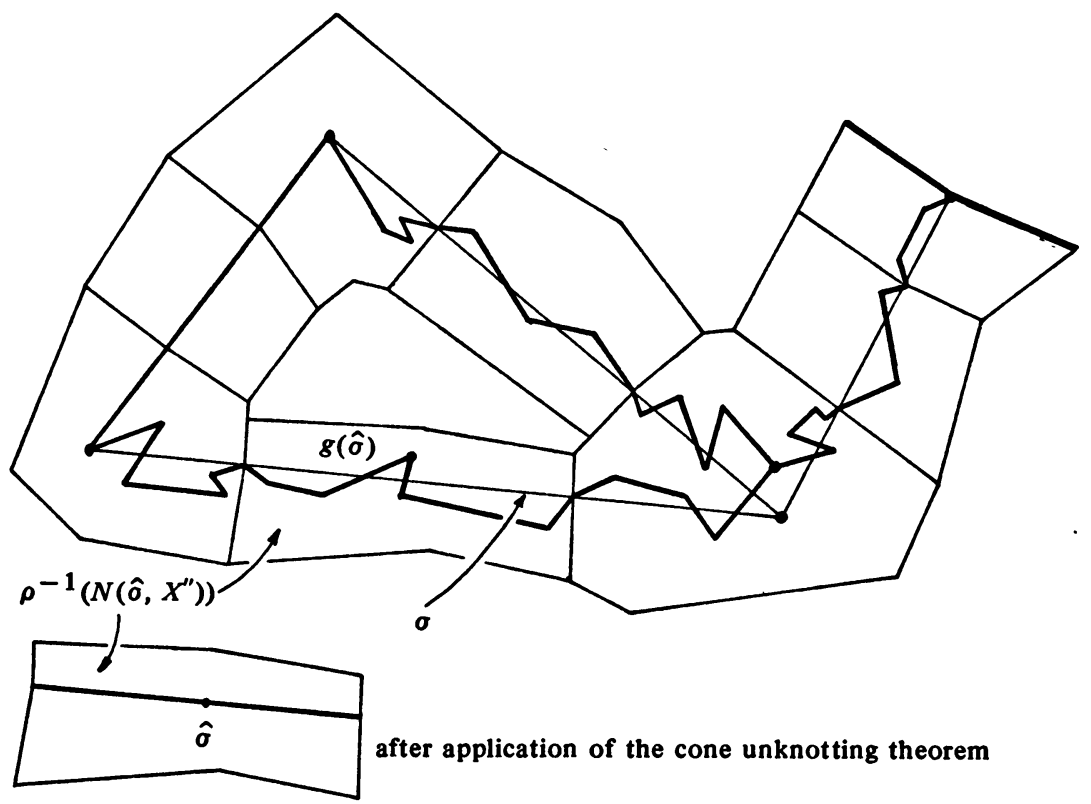

FIGURE 7. The slicing lemma is used in a collar of each block to move $g \mid \bigcup_{\sigma \in X} \partial N\left(\hat{\sigma}, X^{\prime \prime}\right)$ to the inclusion, and at the same time to move $g\left(N\left(\hat{\sigma}, X^{\prime \prime}\right)\right)$ into $\rho^{-1}\left(N\left(\hat{\sigma}, X^{\prime \prime}\right)\right)$ all $\sigma \in X$. The cone unknotting theorem is then applied to move each $g \mid N\left(\hat{\sigma}, X^{\prime \prime}\right)$ to the inclusion in $\rho^{-1}\left(N\left(\hat{\sigma}, X^{\prime \prime}\right)\right)$ fixing $\partial \rho^{-1}\left(N\left(\hat{\sigma}, X^{\prime \prime}\right)\right)$. 
$\omega_{t} \times$ id: $N \times[0,1] \times A \hookleftarrow$. Let $V_{t}=\Omega_{t} \mid(X \times[0,1] \times A) \cup(N \times$ $[0,1] \times B)$ (if $Y \supset(X \cap \partial N)$ let $V_{t}=\Omega_{t}(X \times[0,1] \times A) \cup$ (bdy $N \times[0,1] \times A) \cup(N \times[0,1] \times B))$, and $\widetilde{V}_{t}$ be the close isotopy in $N \times[0,1] \times A$ covering $g \circ V_{t}$. Then

(3) $\Omega_{1} \circ\left(\widetilde{V}_{1}\right)^{-1}\left(g\left(X \times\left[\frac{2 j}{2 w}, 1-\frac{2 j}{2 w}\right] \times A\right)\right) \subset N \times\left[\frac{2 n-\mathrm{i}}{2 w}, 1-\frac{2 j-1}{2 w}\right] \times A$, $\Omega_{1} \circ\left(\widetilde{V}_{1}\right)^{-1}\left(g\left(X \times\left[\frac{2 j}{2 w}, 1-\frac{2 j}{2 w}\right] \times A\right)\right) \cap\left(N \times\left[\frac{2 j+1}{2 w}, 1-\frac{2 j+1}{2 w}\right] \times A\right)$ (4)

$$
=\Omega_{1} \circ\left(\widetilde{V}_{1}\right)^{-1}(g(X \times[0,1] \times A)) \cap\left(N \times\left[\frac{2 j+1}{2 w}, 1-\frac{2 j+1}{2 w}\right] \times A\right) .
$$

If $w$ is chosen sufficiently large, $\Omega_{1} \circ\left(\widetilde{V}_{1}\right)^{-1} \circ \mathrm{g}$ satisfies the hypotheses of Theorem 4 applied to embeddings of $(X \times[0,1]) \times A$ in $(N \times[0,1]) \times A$ that are the identity on $(X \times\{0,1\}) \times A$. The retraction $\rho \times$ id: $N \times[0,1]$ $\rightarrow X \times[0,1]$ makes $N \times[0,1]$ an abstract regular neighborhood of $X \times$ $[0,1]$. Theorem 4 then provides a fiber preserving ambient isotopy $\widetilde{\Psi}_{t}$ of $N \times$ $[0,1] \times I^{k}$ that is fixed on $N \times\{0,1\} \times A$ and such that $\widetilde{\Psi}_{1} \circ \Omega_{1} \circ\left(\widetilde{V}_{1}\right)^{-1}$ $\circ g$ is the identity. Thus, $\Omega_{t} \circ \widetilde{V}_{t}$ followed by $\widetilde{\Psi}_{t}$ is the desired isotopy.

Millett uses his fibered general position to extend Zeeman's sunny collapsing to the fibered case [Mi, §4]. The following lemma is a further extension based on [M1, Lemma 3].

LemMa 17 (SUNNy COllapsing). Suppose $M$ is a manifold and $Y$ is a subcomplex of $M$ with $\operatorname{dim} Y \leqslant \operatorname{dim} M-3$. Let $Z$ be a subcomplex of $Y$ such that each principal simplex of $Y$ not in $Z$ has dimension greater than zero. For sufficiently small $\epsilon>0$, and for arbitrary $\delta>0$, if $f: Y \times J \times I^{k}$ $\rightarrow M \times J \times I^{k}$ is a fiber preserving (but, in general, not level preserving in the $J$ direction) embedding satisfying

(1) $f \mid\left(Z \times J \times I^{k}\right) \cup\left(Y \times J \times \partial I^{k}\right)=$ inclusion,

(2) $f^{-1}\left(\partial(M \times J) \times I^{k}\right) \subset Z \times J \times I^{k}$, and

(3) $\left(\pi_{J} \times \mathrm{id}_{I^{k}}\right) \circ f$ is within $\epsilon$ of $\left(\pi_{J} \times \mathrm{id}_{I^{k}}\right) \circ$ inclusion, it follows that there is a fiber preserving and level preserving in the $J$ direction $\delta$-ambient isotopy $\eta_{t}$ of $M \times J \times I^{k}$ with

(4) $\eta_{t}$ fixed outside the $\delta$-neighborhood of $f\left(Y \times J \times I^{k}\right)$ relative to $f\left(Z \times J \times I^{k}\right)$ and fixed on $M \times(J-(-3, k+1))$

and there is a triangulation of $Y \times J \times I^{k}$ that projects simplicially to a triangulation $K$ of $I^{k}$ and that contains a subcomplex $W$ such that 
(5) $W$ is a subpolyhedron of $Y \times[-4,-1] \times I^{k}$ and $\eta_{1} \circ f\left(W \cap\left((Y-Z) \times J \times I^{k}\right)\right) \subset M \times[-4,-1] \times I^{k}$,

(6) $W \supset\left(\bigcup_{\tau \in \partial K} Y \times[-4,-1] \times \tau\right) \cup\left(\bigcup_{\tau \in K} Z \times[-4,-1] \times \tau\right) \cup$ $\left(Y \times[-4,-3] \times I^{k}\right)$,

(7) the shadow set of $\eta_{1} \circ f(W)$ intersects $\eta_{1} \circ f\left(Y \times J \times I^{k}\right)$ precisely in $\eta_{1}^{\circ} f(W)$,

(8) for each simplex $\tau \in K$, some subdivision of $Y \times[-4,-1] \times \hat{\tau}$ collapses to a subdivision of $W \cap \pi^{-1}(\hat{\tau})$, and

(9) for each simplex $\tau$, the track under the collapse of each point $(y, t, \hat{\tau}) \in Y \times[-4,-1] \times \hat{\tau}$ lies in the $(\epsilon+2 \delta)$-neighborhood of $\{y\} \times$ $J \times\{\hat{\tau}\}$.

Finally, if $L$ is any subdivision of $K$, then the lemma holds with the same $\eta_{t}$ for any triangulation of $Y \times J \times I^{k}$ that projects simplicially to $L$.

One would like Lemma 17 to state that there is a fiber preserving simplicial collapse of $Y \times P_{K}^{-}$to $W$. Unfortunately, such a collapse probably does not exist; at any rate, the proof we have gives fiber preserving cellular collapses, and the usual proof that cellular collapse implies simplicial collapse falls through in the fibered case.

Outline of the proof of Lemma 17. Proceed as in [Mi, §3] to obtain $\eta_{t}$ such that $\pi_{Y \times I^{k}} \circ \eta_{1} \circ f$ is in fibered general position $\operatorname{rel} Z \times J \times I^{k}$. Then subdivide to make $M \times J \times I^{k} \rightarrow M \times I^{k} \rightarrow I^{k}$ simplicial, and again subdivide to make $Y \times J \times I^{k} \rightarrow Y \times I^{k} \rightarrow I^{k}$ simplicial. Let $K$ be the triangulation so obtained on $I^{k}$. It is sufficient to consider the case where $K$ is a single principal simplex, $\Delta^{k}$. Star each simplex in $Y \times J \times \Delta^{k}$ at its barycenter. Build blisters as in [M1, Lemma 3] for all simplexes in the singular set that project onto $\Delta^{k}$, being careful to choose the three points for each blister to lie in $Y \times J \times\left\{\hat{\Delta}^{k}\right\}$. If this is done, the new triangulation of $Y \times J \times \Delta^{s}$ that contains all the blisters will project simplicially to $\left(\partial \Delta^{k}\right) \cdot \hat{\Delta}^{k}$. If the simplexes of $Y \times J \times \Delta^{k}$ that project onto $\Delta^{k}$ are collapsed according to Zeeman, Millett's fibered extension is obtained. If the collapse is done according to Miller, Lemma 17 of this paper is proved. Condition (7) of Lemma 17 results automatically from the fact that the composition of $\eta_{1} \circ f$ with the collapse in $Y \times J \times \Delta^{k}$ is a sunny collapse in $M \times J \times \Delta^{k}$.

Millett pointed out to me the curious case of zero-dimensional principal simplexes. Let $B$ be one. If $k=0$, then $\operatorname{dim}\left(B^{0} \times J\right)=1$ and $\operatorname{dim} M \geqslant 3$, so simple general position makes any collapse along $f\left(B^{0} \times J\right)$ sunny. If $k>$ 0 , however, it is possible to get zero-dimensional singularities in some fibers. Since there is no room in a 1-dimensional set to build blisters, Zeeman's sunny collap- 
sing construction does not apply, and we are stuck with our singularities. This leads to a bit of complication in the slicing lemma which follows. To prepare the way, we remark that in the case $\operatorname{dim} B=0$, we can use the fibered product neighborhood of $f\left(B \times J \times I^{k}\right)$ in $M \times J \times I^{k}$ to extend $f$ to an embedding $Z \times J \times I^{k} \cup(B \times[-a, a]) \times J \times I^{k} \rightarrow M \times J \times I^{k}$ where $a$ is a number such that $f(B \times[-a, a] \times s \times t)$ is contained in an $\epsilon$-neighborhood of $f(B \times\{0\} \times s \times t)$, and such that Lemma 17 holds when $(Z \cup(B \times[-a / 2, a / 2]))$ $\times J \times I^{k}$ is substituted for $(Z \cup B) \times J \times I^{k}$. This is where we slip from codimension 3 to codimension 4 . Under the collapse, $B \times\{0\} \times J \times I^{k}$ may not remain invariant; however, it does stay in $B \times(-a / 4, a / 4) \times J \times I^{k}$.

Let $Y$ be a $(j-1)$-dimensional complex, and $M$ be an $(n-1)$-dimensional abstract regular neighborhood of $Y$ with retraction $\rho: M \rightarrow Y$. Suppose $j \leqslant n-4$. Let $Z$ be a subcomplex of $Y$ that contains its $(j-2)$-skeleton and its intersection with $\partial M$.

Lemma $18(n, j, k)$ (SLicing). For $\epsilon>0$, there is a $\delta>0$ such that if $f$ : $Y \times J \times I^{k} \rightarrow M \times J \times I^{k}$ is a fiber preserving embedding that satisfies

(1) $f \mid\left(Z \times J \times I^{k}\right) \cup\left(Y \times J \times \partial I^{k}\right)=$ inclusion, and

(2) $\left(\rho \times \mathrm{id}_{J} \times \mathrm{id}_{I^{k}}\right) \circ f$ is within $\delta$ of the inclusion, then there is a triangulation $K$ of $I^{k}$ and a fiber preserving ambient isotopy $\Psi_{t}$ of $M \times J \times I^{k}$ satisfying

(3) $\Psi_{t} \mid\left(Z \times J \times I^{k}\right) \cup \partial\left(M \times J \times I^{k}\right)=$ identity,

(4) diameter $\left(\left(\rho \times \mathrm{id}_{I^{k}}\right) \circ \pi_{M \times I^{k}} \circ \Psi_{[0,1]}(q)\right)<\epsilon$, all $q \in M \times J \times I^{k}$,

(5) $\Psi_{1} \circ f \mid Y \times P_{K}=$ identity, and

(6) $\Psi_{1} \circ f\left(Y \times P_{K}^{+}\right) \subset M \times P_{K}^{+}, \Psi_{1} \circ f\left(Y \times P_{K}^{-}\right) \subset M \times P_{K}^{-}$.

Proof. By Theorem $4(n, j-1, k)$, we can assume the extra condition $f \mid Y \times\{l\} \times I^{k}=$ inclusion, all integers $0 \leqslant l \leqslant k$. Use the sunny collapsing lemma to obtain the ambient isotopy $\eta_{t}$ and complex $W$. Since we can choose $\delta$ in that lemma so small that $\eta_{1} \circ f$ also satisfies the hypotheses of the slicing lemma, and since we can choose $\eta_{t}$ to have $M \times\{j\} \times I^{k}$ invariant, we might as well suppose $\eta_{t}=$ identity and $f\left(Y \times\{l\} \times I^{k}\right) \subset M \times\{l\} \times I^{k}$, all integers $0 \leqslant l \leqslant k$. If $Y-Z$ has zero-dimensional principal simplexes, we might as well suppose there is just one, and make the modification described above.

Suppose $M \times\{l\} \times I^{k}$ and $Y \times\{l\} \times I^{k}$ are subcomplexes of $M \times J \times$ $I^{k}$ and $Y \times J \times I^{k}$ for integers $-4 \leqslant l \leqslant k+1$. Subdivide $M \times J \times I^{k}, Y$ $\times J \times I^{k}$, and $I^{k}$ to obtain triangulations $Q, L$, and $K$ on which $f, \pi_{M \times I^{k}}$, and $\pi$ are simplicial. Let

$$
\begin{gathered}
V=Q \cap\left(\left(\bigcup_{\tau \in K} \partial M \times[-4,-1] \times \tau\right) \cup\left(\bigcup_{\tau \in \partial K} M \times[-4,-1] \times \tau\right)\right. \\
\cup\left(M \times[-4,-3] \times I^{k} \cup(\text { shadow set of } f(W))\right)
\end{gathered}
$$


Then for each $\tau \in K, M \times[-4,-1] \times\{\hat{\tau}\}$ collapses to $V \cap(M \times[-4,-1]$ $\times\{\hat{\gamma}\})$ in a way that leaves $\gamma \times J \times I^{k}$ invariant for each simplex $\gamma \in M$. Special uniqueness therefore yields a fiber preserving ambient isotopy $\Psi_{t}^{I}$ of $M \times J \times I^{k}$ with

(7) $\Psi_{1}^{\mathrm{l}}\left(M \times P_{K}^{-}\right)=N\left(V, Q^{\prime \prime}\right)$,

(8) diameter $\left(\pi_{M \times I^{k}} \circ \Psi_{[0,1]}^{\mathrm{I}}(q)\right)$ is small if $\delta$ is small.

For the next step, if there are no zero-dimensional principal simplexes, we again apply special uniqueness to the collapse of $U_{\tau \in K} Y \times[-4,-1] \times \tau$ to $W$, to construct a fiber preserving ambient isotopy $\Phi_{t}$ in $Y \times J \times I^{k}$ that satisfies

(9) $\Phi_{t}\left(Y \times P_{K}^{-}\right)=N\left(W, L^{\prime \prime}\right)$,

(10) diameter $\left(\pi_{M \times I^{k}}{ }^{\circ} \Phi_{[0,1]}(l)\right)$ is small if $\delta$ is small,

(11) $\Phi_{t} \mid\left(Y \times(J-(-3, k+1)) \times I^{k}\right)=$ identity, and

(12) $\Phi_{t}$ leaves each simplex $\gamma \in Y$ invariant.

Since the triangulation $L^{\prime \prime} \cap\left(Z \times J \times I^{k}\right)$ is induced from $Q^{\prime \prime}$ by the inclusion map, we can have also

(13) $\Phi_{t}\left|Z \times J \times I^{k}=\Psi_{t}^{\mathrm{I}}\right| Z \times J \times I^{k}$.

By (11), (12), and (13), Corollary 15 provides an isotopy $\Psi_{t}^{\mathrm{II}}$ covering $f \circ \Phi_{t}$ in $M \times J \times I^{k}$ such that

(14) $\Psi_{t}^{\mathrm{II}}\left|\partial\left(M \times J \times I^{k}\right) \cup\left(Z \times J \times I^{k}\right)=\Psi_{t}^{\mathrm{I}}\right| \partial\left(M \times J \times I^{k}\right) \cup Z \times J \times$ $I^{k}$.

By (10) together with (4) and (6) of Corollary 15 , (2) of the slicing lemma, and (9) of the sunny collapsing lemma,

(15) diameter $\left(\rho \times \mathrm{id}_{I^{k}} \circ \pi_{M \times I^{k}} \circ \Psi_{[0,1]}^{\mathrm{II}}(q)\right)$ is small if $\delta$ is small, all $q \in M \times J \times I^{k}$.

Define $\Psi_{t}^{\mathrm{III}}=\left(\Psi_{t}^{\mathrm{I}}\right)^{-1} \circ \Psi_{t}^{\mathrm{II}}$. Then, from (14), we have

(16) $\Psi_{t}^{\mathrm{III}} \mid \partial\left(M \times J \times I^{k}\right) \cup\left(Z \times J \times I^{k}\right)=$ identity.

From (8) and (15) we have $M \times J \times I^{k}$.

(17) diameter $\left(\rho \times \mathrm{id}_{I^{k}} \circ \pi_{M \times I} k^{\circ} \psi_{[0,1]}^{\mathrm{II}}(q)\right)$ is small if $\delta$ is small, $q \in$

Finally, recall that the shadow set of $f(W)$ intersects $f\left(Y \times J \times I^{k}\right)$ only in $f(W)$. This provides the punch line:

$$
\begin{gathered}
f^{-1}\left(N\left(V, Q^{\prime \prime}\right)\right)=N\left(W, L^{\prime \prime}\right) \text { and } \\
f^{-1}\left(\text { frontier } N\left(V, Q^{\prime \prime}\right) \text { in } Q\right)=\text { frontier } N\left(W, L^{\prime \prime}\right) \text { in } L .
\end{gathered}
$$

It follows from (7) and (9) that

$$
\Psi_{1}^{\mathrm{III}} \circ f: Y \times\left(P_{K}^{+}, P_{K}^{-} ; P_{K}\right) \rightarrow M \times\left(P_{K}^{+}, P_{K}^{-} ; P_{K}\right)
$$

is a map of triads. 
In case $B$ is a zero-dimensional principal simplex, collapse

$$
\bigcup_{\tau \in K}(Z \cup(B \times[-a, a])) \times[-4,-1] \times \tau
$$

to

$$
\begin{aligned}
\left(\bigcup_{\tau \in K}\left(Z \cup\left(B \times\left(\{-a, a\} \cup\left[\frac{-a}{2}, \frac{a}{2}\right]\right)\right)\right) \times[-4,-1] \times \tau\right) \\
\cup\left(B \times[-a, a] \times[-4,-3] \times I^{k}\right) .
\end{aligned}
$$

Recall that $W$ is the complex assigned to

$$
\bigcup_{\tau \in K}(Z \cup(B \times[-a / 2, a / 2])) \times[-4,-1] \times \tau
$$

by the sunny collapsing lemma. Let

$$
\begin{gathered}
\widetilde{W}=W \cup\left(\bigcup_{\tau \in K}(B \times\{-a, a\}) \times[-4,-1] \times \tau\right) \\
\cup\left(B \times[-a, a] \times[-4,-3] \times I^{k}\right) .
\end{gathered}
$$

Apply special uniqueness to $\widetilde{W}$ in $(Z \cup(B \times[-a, a])) \times J \times I^{k}$ to obtain an isotopy $\Phi_{t}$ of $(Z \cup(B \times[-a, a])) \times J \times I^{k}$. By the discussion after Lemma 17 and condition (3) of special uniqueness we can assume

$$
\Phi_{t}\left(B \times\{0\} \times J \times I^{k}\right) \subset B \times[-a / 2, a / 2] \times J \times I^{k}
$$

and

$\Phi_{1}\left((Z \cup(B \times[-a, a])) \times P_{K}^{-}\right)=N\left(\tilde{W}, L^{\prime \prime}\right)$.

Thus,

$\Phi_{1}\left(B \times\{0\} \times J \times I^{k}\right) \cap N\left(\tilde{W}, L^{\prime \prime}\right)$

$$
\begin{aligned}
& =\Phi_{1}\left(B \times\{0\} \times J \times I^{k}\right) \cap N\left(W, L^{\prime \prime} \cap\left(Z \cup\left(B \times\left[\frac{-a}{2}, \frac{a}{2}\right]\right)\right) \times J \times I^{k}\right) \\
& =\Phi_{1}\left((B \times\{0\}) \times P_{K}^{-}\right)
\end{aligned}
$$

and

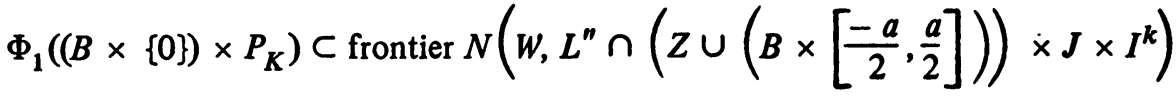

$$
\text { in }\left(Z \cup\left(B \times\left[\frac{-a}{2}, \frac{a}{2}\right]\right)\right) \times J \times I^{k} \text {. }
$$

Define $\Psi_{t}^{\text {II }}$ and $\Psi_{t}^{\text {III }}$ as before. $\Psi_{t}^{\text {III }}$ then satisfies (16) and (17). Since we 
have for sufficiently small $\delta$ that the shadow set of $f(W)$ intersects $f\left((Z \cup(B \times[-a / 2, a / 2])) \times J \times I^{k}\right)$ in precisely $f(W)$ it follows that

$$
\begin{aligned}
\left((Z \cup(B \times[-a / 2, a / 2])) \times J \times I^{k}\right) \cap f^{-1}\left(N\left(V, Q^{\prime \prime}\right)\right) \\
=N\left(W, L^{\prime \prime} \cap\left((Z \cup(B \times[-a / 2, a / 2])) \times J \times I^{k}\right)\right)
\end{aligned}
$$

and

$$
\begin{aligned}
\left((Z \cup(B \times[-a / 2, a / 2])) \times J \times I^{k}\right) \cap f^{-1}\left(\text { frontier } N\left(V, Q^{\prime \prime}\right) \text { in } Q\right) \\
=\text { frontier } N\left(W, L^{\prime \prime} \cap(Z \cup(B \times[-a / 2, a / 2])) \times J \times I^{k}\right) \\
\\
\text { in }(Z \cup(B \times[-a / 2, a / 2])) \times J \times I^{k} .
\end{aligned}
$$

Together with (19) this implies $\Psi_{1}^{\text {III }}$ is the desired map of triads.

Let $g \equiv \Psi_{1}^{\mathrm{III}} \circ f$. It remains to move $g \mid Y \times P_{K}$ to the identity. Define $p_{K}=\bigcup_{\sigma \in K}\{k-\operatorname{dim} \sigma\} \times N\left(\hat{\sigma}, K^{\prime \prime}\right)$. By Theorem $4(n-1)$, there is a fiber preserving ambient isotopy $\psi_{t}$ of $M \times p_{K}$ satisfying

(20) $\psi_{1} \circ g \mid Y \times p_{K}=$ identity,

(21) $\psi_{t}^{\circ} g \mid\left((Z \cup \partial M) \times p_{K}\right) \cup\left(Y \times\left(p_{K} \cap\left(J \times \partial I^{k}\right)\right)\right)=$ identity, and

(22) diameter $\left(\rho \times \mathrm{id}_{I^{k}}\right) \circ \pi_{M \times I^{k}} \circ \psi_{[0,1]}(q)$ is small if $\delta$ is small, all $q \in M \times p_{K}$.

Now $M \times p_{K}$ is collared in $M^{*}=M \times\left(\bigcup_{j=0}^{k}\{j\}\right) \times I^{k}$, and we can use the collar to extend $\psi_{t}$ to a fiber preserving ambient isotopy in $M^{*}$ that is the identity outside the collar. The extension satisfies (20)-(22). Again, $M^{*}$ has a fiber preserving two-sided collar in $M \times J \times I^{k}$, and we can use this collar to further extend $\psi_{t}$ to a fiber preserving ambient isotopy (which we call $\Psi_{t}^{\text {IV }}$ ) of $M \times J \times I^{k}$, that satisfies (20)-(22) when $\Psi_{t}^{\mathrm{IV}}$ is substituted for $\psi_{t}$, and that leaves $M \times P_{K}$ invariant.

Denote by $P_{K}^{j}$ the union over simplexes $\sigma \in K^{\prime}, \operatorname{dim} \sigma=k-j$, of the collection of closures of the components of $\left(P_{K}-p_{K}\right) \cap\left(D\left(\sigma, K^{\prime \prime}\right) \times J\right)$. Observe that if $\alpha$ is an element of $P_{K}^{j}$, then $\Psi_{1}^{\mathrm{IV}} \circ g(Y \times \alpha) \subset M \times \alpha$ and $\Psi_{1}^{\mathrm{IV}} \circ g \mid \alpha \cap p_{K}=$ identity. By induction on increasing $j$, use the addendum to the equivalence theorem to construct, for each $\alpha \in P_{K}^{j}$, isotopies of $M \times \alpha$. Since for a given $j$ their support is disjoint, they fit together to give an isotopy $\Psi_{t}^{\mathrm{V}}$ of $M \times J \times\left(\right.$ dual $j$-skeleton of $\left.K^{\prime \prime}\right)$ satisfying

(23) $\Psi_{1}^{\mathrm{V}, j} \circ \Psi_{1}^{\mathrm{V}, j-1} \circ \ldots \circ \Psi_{1}^{\mathrm{V}, 0} \circ \Psi_{1}^{\mathrm{IV}} \circ g=$ identity.

(24) $\Psi_{t}^{V, j}$ is the identity on the complement of $M \times P_{K}^{j}$ in $M \times J \times$ (dual $j$-skeleton of $\left.K^{\prime}\right)$ and on $M \times J \times\left(\right.$ dual $(j-1)$-skeleton of $\left.K^{\prime}\right)$,

(25) $\Psi_{t}^{\mathrm{V}, j} \mid\left((Z \cup \partial M) \times P_{K}^{j}\right) \cup\left(Y \times\left(P_{K}^{j} \cap\left(J \times \partial I^{k}\right)\right)\right)=$ identity,

(26) diameter $\left(\left(\rho \times \mathrm{id}_{I^{k}}\right) \circ \pi_{M \times I^{k}} \circ \Psi_{[0,1]}^{\mathrm{V}, j}(q)\right)$ is small if $\delta$ is small, all $q \in M \times J \times\left(\right.$ dual $j$-skeleton of $\left.K^{\prime \prime}\right)$. 
Use a fiber preserving collar of $M \times J \times$ (dual $j$-skeleton) in $M \times J \times I^{k}$ to extend $\Psi_{t}^{V, j}$ to a fiber preserving isotopy fixed on $\left(M \cup p_{K}\right) \cup((Z \cup \partial M)$ $\left.\times J \times I^{k}\right) \cup\left(Y \times J \times \partial I^{k}\right)$. Define $\Psi_{t}^{\mathrm{V}}=\Psi_{t}^{\mathrm{V}, k} \circ \cdots \circ \Psi_{t}^{\mathrm{V}, 0}$. Finally define $\Psi_{t}=\Psi_{t}^{\mathrm{V}} \circ \Psi_{t}^{\mathrm{IV}} \circ \Psi_{t}^{\mathrm{III}}$ to complete the proof of the slicing lemma.

LEMmA $19(n, j, k, l)$. Let $X$ be a complex of dimension $j$, and let $Y$ be a subcomplex of $X$ that contains the $(j-1)$-skeleton. Let $\widetilde{X}$ be a triangulation of $X$. Then for $\epsilon>0$, there is a $\delta>0$ such that if $(N, M, \tilde{\rho})$ is an abstract regular neighborhood of $X$ with $Y \supset(\partial N \cap X)$ and with $\tilde{\rho}^{-1}\left(D\left(\sigma, X^{\prime \prime}\right)\right)$ an $(n-\operatorname{dim} \sigma)$-ball for each simplex $\sigma \in \tilde{X}^{\prime}$, and if $g: X \times I^{k} \rightarrow N \times I^{k}$ is a fiber preserving embedding with

(1) $g^{-1}\left(\partial N \times I^{k}\right)=(X \cap \partial N) \times I^{k}$,

(2) $g \mid X \times \partial I^{k} \cup Y \times I^{k}=$ inclusion,

(3) $\left(\tilde{\rho} \times \mathrm{id}_{I^{k}}\right) \circ g$ is within $\delta$ of the inclusion, and also satisfying for each simplex $\tau \in \tilde{X}$ with $\operatorname{dim} \tau \leqslant l-1$

(4) $g \mid \partial N\left(\hat{\tau}, \widetilde{X}^{\prime \prime}\right) \times I^{k}=$ inclusion,

(5) $g\left(N\left(\hat{\tau}, \widetilde{X}^{\prime \prime}\right) \times I^{k}\right)=g\left(\widetilde{X} \times I^{k}\right) \cap \tilde{\rho}^{-1}\left(N\left(\hat{\tau}, \tilde{X}^{\prime \prime}\right)\right) \times I^{k}$,

it follows that there is a triangulation $K_{l}$ of $I^{k}$ and a fiber preserving ambient isotopy $\Theta_{t}^{l}$ of $N \times I^{k}$ such that

(6) $\Theta_{t}^{l} \mid\left((Y \cup \partial N) \times I^{k}\right) \cup\left(N \times \partial I^{k}\right)=$ identity,

(7) $\Theta_{1}^{l} \circ g \mid X \times L_{l}=$ inclusion, where $L_{l}$ is the $(k-1)$ dual skeleton of $K_{l}^{\prime}$ in $K_{l}^{\prime \prime}$, and for each simplex $\mu \in K_{l}$ there is a homeomorphism $h_{\mu}: X \rightarrow$ $X$ close to the identity such that

(8) $h_{\mu}\left(N\left(\hat{\tau}, \widetilde{X}^{\prime \prime}\right)\right)=N\left(\hat{\tau}, \widetilde{X}^{\prime \prime}\right)$ for each simplex $\tau \in \tilde{X}$, with $\operatorname{dim} \tau \leqslant l-1$, and for each simplex $\tau \in \tilde{X}$, with $\operatorname{dim} \tau \leqslant l$,

(9) $\Theta_{1}^{l} \circ g \mid h_{\mu}\left(\partial N\left(\hat{\tau}, \widetilde{X}^{\prime \prime}\right)\right) \times N\left(\hat{\mu}, K_{l}^{\prime \prime}\right)=$ inclusion, and

(10) $\Theta_{1}^{l} \circ g\left(h_{\mu}\left(N\left(\hat{\tau}, \tilde{X}^{\prime \prime}\right)\right)\right) \times N\left(\hat{\mu}, K_{l}^{\prime \prime}\right)=\Theta_{1}^{l} \circ g\left(X \times N\left(\hat{\mu}, K_{l}^{\prime \prime}\right)\right) \cap$ $h_{\mu}\left(N\left(\hat{\tau}, \widetilde{X}^{\prime \prime}\right)\right) \times N\left(\hat{\mu}, K_{l}^{\prime \prime}\right)$.

In addition,

(11) diameter $\left(\left(\rho \times \mathrm{id}_{I^{k}}\right) \circ \Theta_{[0,1]}^{l}(\mu)\right)$ is small if $\delta$ is small, all points $\mu \in N \times I^{k}$.

Proof. Choose a small two-sided collar neighborhood of

$$
\begin{gathered}
U=\bigcup_{\substack{l \text { simplexes } \\
\mu \text { in } \tilde{X}}} \partial N\left(\hat{\mu}, X^{\prime \prime}\right)-\bigcup_{\text {simplexes } \tau} N\left(\hat{\tau}, X^{\prime \prime}\right) \\
\text { in } \tilde{X} \text { if } \operatorname{dim} \leqslant l-1
\end{gathered}
$$

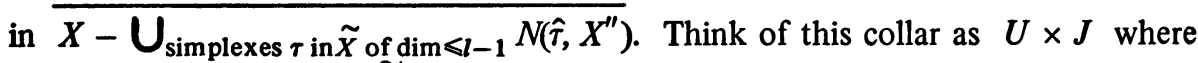
$U \times\{0\}=U$. Let $Q=\tilde{\rho}^{-1}(U)$. By Proposition 5 we may assume that 
$(Q, \tilde{\rho} \mid Q)$ is an abstract regular neighborhood of $U$, and in fact, $\tilde{\rho}^{-1}(U \times J)=$ $Q \times J$. After an application of Corollary 15, we can assume $g\left(U \times J \times I^{k}\right) \subset$ $Q \times J \times I^{k}$. Then if we set $F=g \mid U \times J \times I^{k}$, we can apply the slicing lemma to find a triangulation $K_{l}$ of $I^{k}$ and an ambient isotopy $\Theta_{t}^{l, I}$. For each simplex $\mu$ in $K_{l}$ choose a homeomorphism $h_{\mu}$, preserving simplexes of $\tilde{X}$, such that $h_{\mu}\left(N\left(\hat{\tau}, \tilde{X}^{\prime \prime}\right)=N\left(\hat{\tau}, \tilde{X}^{\prime \prime}\right)\right.$ for each $\tau \in \tilde{X}$ with $\operatorname{dim} \tau \leqslant l-1$, and

$$
\begin{gathered}
h_{\mu}\left(\partial N\left(\sigma, X^{\prime \prime}\right)-\underset{\text { simplexes } \tau}{\bigcup} N\left(\hat{\tau}, \widetilde{X}^{\prime \prime}\right)\right) \\
\text { in } \tilde{X} \text { of } \operatorname{dim}<l-1 \\
=U \times\{k-\operatorname{dim} \mu\} \subset U \times J
\end{gathered}
$$

for each $l$-simplex $\sigma \in \tilde{X}$.

Condition (11) follows from (4) of the slicing lemma together with the fact that we chose the collar $Y \times J$ of $Y$ to be small in the $J$ direction.

We still have to arrange (7) of Lemma 19. But this we do with the equivalence theorem, using $L_{l}$ as the index set. (Recall that $\operatorname{dim} L_{l}=k-1$.) This yields a small fiber preserving ambient isotopy of $N \times L_{l}$, fixed on

$$
\begin{gathered}
\left(Q \times\left(P_{K_{l}} \cap\left(J \times L_{l}\right)\right)\right) \cup\left(\left(Y \cup \underset{\tau \in \tilde{X}}{\cup} \tilde{\rho}^{-1}\left(N\left(\hat{\tau}, \tilde{X}^{\prime \prime}\right)\right)\right) \times L_{l}\right) \\
\cup\left(N \times\left(\partial I^{k} \cap L_{l}\right)\right) \cup\left(\partial N \times L_{l}\right)
\end{gathered}
$$

for simplexes $\tau \in \tilde{X}, \operatorname{dim} \tau \leqslant l-1$.

Let $\Theta_{t}^{L, I I}$ be a small fiber preserving ambient isotopy of $N \times I^{k}$ that extends the isotopy on $N \times L_{l}$, and is the identity on

$$
\left(Q \times P_{K_{l}}\right) \cup\left(\left(Y \cup \bigcup_{\tau \in \tilde{X}} \tilde{\rho}^{-1}\left(N\left(\hat{\tau}, \tilde{X}^{\prime \prime}\right)\right)\right) \times I^{k}\right) \cup\left(N \times \partial I^{k}\right) \cup\left(\partial N \times I^{k}\right)
$$

for simplexes $\tau \in \tilde{X}, \operatorname{dim} \tau \leqslant l-1$. Set $\Theta_{t}^{l}=\Theta_{t}^{l, \mathrm{II}} \circ \Theta_{t}^{l, 1}$.

PROOF OF THE EQUIVALENCE THEOREM. By application of Lemma $19(n$, $j, k, 1)$ through Lemma $19(n, j, k, j), I^{k}=\bigcup_{\alpha} D_{\alpha}$, where $D_{\alpha}$ is a $k$-ball and $D_{\alpha} \cap D_{\beta} \subset \partial D_{\alpha} \cap \partial D_{\beta}$, and

$$
g \mid\left(X \times\left(\bigcup_{\alpha} \dot{\partial} D_{\alpha}\right)\right) \cup\left(Y \times I^{k}\right)=\text { identity. }
$$

For each $\alpha$, there is a small homeomorphism $h_{\alpha}: X \rightarrow X$ such that

$$
g \mid\left(\bigcup_{\text {simplexes } \tau \text { in } X} h_{\alpha}\left(\partial N\left(\hat{\tau}, \tilde{X}^{\prime \prime}\right) \times D_{\alpha}\right)\right)=\text { inclusion, and }
$$




$$
g\left(h_{\alpha}\left(N\left(\hat{\tau}, \tilde{X}^{\prime \prime}\right) \times D_{\alpha}\right)\right)=g\left(X \times D_{\alpha}\right) \cap\left(\tilde{\rho}^{-1}\left(h_{\alpha}\left(N\left(\hat{\tau}, \tilde{X}^{\prime \prime}\right)\right) \times D_{\alpha}\right)\right),
$$

all simplexes $\tau \in \tilde{X}$.

We can now apply the fibered version of the cone unknotting theorem to

$$
g \mid h_{\alpha}\left(N\left(\hat{\tau}, \tilde{X}^{\prime \prime}\right)\right) \times D_{\alpha}: h_{\alpha}\left(N\left(\hat{\tau}, \tilde{X}^{\prime \prime}\right)\right) \times D_{\alpha} \rightarrow \tilde{\rho}^{-1}\left(h_{\alpha}(N(\hat{\tau}, \tilde{X}))\right) \times D_{\alpha}
$$

to obtain a fiber preserving ambient isotopy $\Theta_{t}^{\tau, \alpha}$ of $\tilde{\rho}^{-1}\left(h_{\alpha}\left(N\left(\hat{\tau}, \tilde{X}^{\prime \prime}\right)\right)\right) \times D_{\alpha}$ satisfying

(4) $\Theta_{t}^{\tau, \alpha} \mid h_{\alpha}\left(\partial\left(\tilde{\rho}^{-1}\left(N\left(\hat{\tau}, \tilde{X}^{\prime \prime}\right)\right)\right)\right) \times D_{\alpha} \cup\left(Y \cap h_{\alpha}\left(N\left(\tau, \tilde{X}^{\prime \prime}\right)\right)\right) \times D_{\alpha}=$ identity,

$$
\Theta_{t}^{\tau, \alpha} \circ g\left(h_{\alpha}\left(N\left(\hat{\tau}, \tilde{X}^{\prime \prime}\right)\right) \times D_{\alpha}\right)=\text { inclusion. }
$$

Extend $\Theta_{t}^{\tau, \alpha}$ by the identity to obtain a fiber preserving ambient isotopy, also called $\Theta_{t}^{\tau, \alpha}$, of $N \times I^{k}$. Define $\Theta_{t}=$ composition $_{\tau, \alpha} \Theta_{t}^{\tau, \alpha}$. Since

$$
\operatorname{int}\left(\tilde{\rho}^{-1}\left(h_{\alpha}\left(N\left(\hat{\tau}, \tilde{X}^{\prime \prime}\right)\right)\right) \times D_{\alpha}\right) \cap \operatorname{int}\left(\tilde{\rho}^{-1}\left(h_{\beta}\left(N\left(\hat{\mathcal{V}}, \tilde{X}^{\prime \prime}\right)\right)\right) \times D_{\beta}\right)=\varnothing
$$

unless $\tau=\nu$ and $\alpha=\beta$, the order of the composition is irrelevant. Since we can choose $h_{\alpha}$ as close to the identity as we like, we can arrange that $h_{\alpha}\left(N\left(\hat{\tau}, \widetilde{X}^{\prime \prime}\right)\right) \subset N\left(N\left(\hat{\tau}, \widetilde{X}^{\prime \prime}\right), X^{\prime \prime}\right)$, and so satisfy $(6)$ of Theorem $6(n, j, k)$. Finally, it is clear that $\Theta_{1} \circ g=$ inclusion and

$$
\Theta_{t} \mid N \times \partial I^{k} \cup(Y \cup \partial N) \times I^{k}=\text { identity } .
$$

This completes the proof of Theorem $6(n, j, k)$.

To prove Theorem $4(n)$ from Theorem $6(n)$, choose a triangulation $\tilde{N}$ of $N$ which retracts simplicially to a triangulation $\widetilde{X}$ of $X$ where the mesh of $\tilde{X}$ is less than $\epsilon / 3$. Then, since diameter $N\left(N\left(\hat{\tau}, \widetilde{X}^{\prime \prime}\right), \widetilde{X}^{\prime \prime}\right)<\epsilon$, Theorem $4(n)$ follows immediately.

\section{REFERENCES}

[B] R. H. Bing, Radial engulfing, Conference on the Topology of Manifolds (Michigan State Univ., E. Lansing, Mich., 1967), Prindle, Weber \& Schmidt, Boston, Mass., 1968, pp. 1-18. MR 38 \#6560.

[Bro] W. Browder, Open and closed disk bundles, Ann. of Math. (2) 83 (1966), 218230. MR 32 \#6485.

[Br] J. L. Bryant, Approximating close embeddings of polyhedra in codimension three, Trans. Amer. Math. Soc. 170 (1972), 85-95.

[B \& S] J. L. Bryant and C. L. Seebeck, Locally nice embeddings in codimension three, Quart. J. Math. Oxford Ser. (2) 21 (1970), 265-272. MR 44 \#7560. 
[C] M. M. Cohen, A general theory of relative regular neighborhoods, Trans. Amer. Math. Soc. 136 (1969), 189-229. MR 40 \#2052.

[Co] R. Connelly, Unknotting close polyhedra in codimension three, Topology of Manifolds (Proc. Inst., Univ. of Georgia, Athens, Ga., 1969), Markham, Chicago, Ill., 1970, pp. 384-388. MR 43 \#2712.

[E] R. D. Edwards, The equivalence of close piecewise linear embeddings, General Topology and Appl. (to appear).

[E \& W] S. Eilenberg and R. L. Wilder, Uniform local connectedness and contractibility, Amer. J. Math. 64 (1942), 613-622. MR 4, 87.

[H] J. F. P. Hudson, Extending piecewise-linear isotopies, Proc. London Math. Soc. (3) 16 (1966), 651-668. MR 34 \#2020.

[L] W. B. R. Lickorish, Piecewise linear unknotting of cones, Topology 4 (1965), 6791. MR 34 \#3585.

[Lu] E. Lusk, Homotopy groups of spaces of embeddings, Thesis, University of Maryland, 1970; announced in Notices Amer. Math. Soc. 17 (1970), 527. Abstract \#674-23.

[M1] R. T. Miller, Close isotopies on piecewise-linear manifolds, Trans. Amer. Math. Soc. 151 (1970), 597-628. MR 42 \#1 127.

[M2] - Approximating codimension 3 embeddings, Ann. of Math. (2) 95 (1972), 406-416.

[Mi] K. C. Millett, Piecewise linear concordances and isotopies, Mem. Amer. Math. Soc., no. 153, 1974.

[Z] E. C. Zeeman, Seminar on combinatorial topology, Inst. Hautes Études. Sci., Paris, 1963 (mimeographed notes).

\section{DEPARTMENT OF MATHEMATICS, MICHIGAN STATE UNIVERSITY, EAST LANSING, MICHIGAN 48823}

Current address: Department of Mathematics, University of California, Berkeley, California 94720 